\title{
MINIMAL HYPERSURFACES WITH THREE PRINCIPAL CURVATURE FIELDS IN $\mathbf{S}^{n+1}$
}

\author{
By Tominosuke Otsuki
}

As is well known, there are many works on minimal hypersurfaces with two regular principal curvature fields in the space forms, especially the spheres. On the contrary, it seems we have very few works on minimal hypersurfaces with more than two principal curvature fields in such spaces. In the present paper, we shall study minimal hypersurfaces in $S^{n+1}$ which have three regular and nonsimple principal curvature fields, say $\mu_{1}, \mu_{2}$ and $\mu_{3}$.

In $\S 1$, we shall state some fundamental theorems in the following argument. In $\S 2$ and $\S 3$, we shall develope a general theory on such hypersurfaces and find that the three tangent vector fields $H\left(\mu_{i}\right)$ (defined by (3.13)) corresponding to $\mu_{i}, i=1,2,3$, play important role in our investigation. In $\S 4$ and $\S 5$, we shall treat the case in which one of $H\left(\mu_{\imath}\right)$ vanishes identically and give an example of such hypersurfaces. Finally, we shall investigate the case in which $H\left(\mu_{2}\right)$ $\not \equiv 0, i=1,2,3$, and show that each $\mu_{\imath}$ can not be constant (Theorem 5), which tells us that in order to construct examples of such hypersurfaces each $\mu_{\imath}$ must be considered as a nonconstant function.

\section{$\S 1$. Preliminaries}

Let $M=M^{n}$ be a hypersurface in an $(n+1)$-dimensional Riemannian manifold $\bar{M}=\bar{M}^{n+1}$ of constant curvature $\bar{c}$. Let $\bar{\omega}_{A}, \bar{\omega}_{A B}=-\bar{\omega}_{B A}, A, B=1,2, \cdots, n+1$, be the basic and connection forms of $\bar{M}$ on the orthonormal frame bundle $F(\bar{M})$ over $\bar{M}$, which satisfy the structure equations

$$
d \bar{\omega}_{A}=\sum_{B} \bar{\omega}_{A B} \wedge \bar{\omega}_{B}, \quad d \bar{\omega}_{A B}=\sum_{C} \bar{\omega}_{A C} \wedge \bar{\omega}_{C B}-\bar{c} \bar{\omega}_{A} \wedge \bar{\omega}_{B}
$$

Let $B$ be the submanifold of $F(\bar{M})$ over $M$ composed of $b=\left(x, e_{1}, \cdots, e_{n+1}\right)$ such that $\left(x, e_{1}, \cdots, e_{n}\right) \in F(M)$, where $F(M)$ is the orthonormal frame bundle of $M$ with the induced Riemannian metric from $\bar{M}$. Then, deleting the bars of $\bar{\omega}_{A}, \bar{\omega}_{A B}$ on $B$, we have

$$
\begin{gathered}
\omega_{n+1}=0, \quad \omega_{i(n+1)}=\sum_{\jmath} A_{\imath \jmath} \omega_{\jmath}, \quad A_{\imath j}=A_{j i}, \\
i, \jmath=1,2, \cdots, n .
\end{gathered}
$$

Recieved January 19, 1976 
Let $\Phi(\omega):=\sum_{i, j} A_{\imath j} \omega_{i} \omega_{\jmath}$ and $\Psi(\omega):=\sum_{\imath, j, k} B_{i j k} \omega_{i} \omega_{j} \omega_{k}$ be the 2nd and 3rd fundamental forms of $M$ respectively, where $B_{\imath j k}$ are defined by

$$
D A_{\imath \jmath}:=d A_{\imath \jmath}-\sum_{k} \omega_{\imath k} A_{k j}-\sum_{k} \omega_{j k} A_{i k}=\sum_{k} B_{i j k} \omega_{k}
$$

and

$$
B_{\imath j k}=B_{j i k}=B_{i k \jmath} .
$$

Now, let $k$ be a principal curvature of $M$ at $x \in M$, an eigen value of $\Phi$ at $x$, and denote the tangent subspace of all principal tangent vectors for $k$ and the zero vector at $x$ by $E(k, x) . \operatorname{dim} E(k, x)$ is equal to the multiplicity of $k$.

Let $\mu$ be a smooth principal curvature field of $M$. If $E(\mu, x)=E(\mu(x), x)$ is of constant dimension, then $E(\mu, x), x \in M$, make a smooth distribution of $M$, which we denote by $E(\mu)$. We call such field $\mu$ regular. We have the following theorem.

TheOREM A. Let $M$ be a hypersurface immersed in an $(n+1)$-dimensional Riemannian manifold $\bar{M}$ of constant curvature $\bar{c}$ and suppose that $M$ has a regular principal curvature field $\mu$, then the distribution $E(\mu)$ is completely integrable.

Proof. Let $m$ be the dimension of the distribution of $E(\mu)$ and take only the frames $b$ such that $e_{\alpha} \in E(\mu), \alpha=1,2, \cdots, m$. Then, we have

$$
\omega_{\alpha(n+1)}=\mu \omega_{\alpha}, \quad \omega_{r(n+1)}=\sum_{t} A_{r t} \omega_{t} .
$$

In the proof, we suppose the ranges of indexes as follows:

$$
\alpha, \beta, \cdots=1,2, \cdots, m ; \quad r, t, \cdots=m+1, \cdots, n .
$$

From (1.1) and (1.5), we get easily

$$
\begin{aligned}
d \omega_{\alpha(n+1)} & =d \mu \wedge \omega_{\alpha}+\mu\left(\sum_{\beta} \omega_{\beta} \wedge \omega_{\beta \alpha}+\sum_{r} \omega_{r} \wedge \omega_{r \alpha}\right), \\
d \omega_{\alpha(n+1)} & =\sum_{\beta} \omega_{\alpha \beta} \wedge \omega_{\beta(n+1)}+\sum_{r} \omega_{\alpha r} \wedge \omega_{r(n+1)} \\
& =\mu \sum_{\beta} \omega_{\beta} \wedge \omega_{\beta \alpha}-\sum_{r, t} A_{r t} \omega_{t} \wedge \omega_{\alpha r}
\end{aligned}
$$

and hence

$$
d \mu \wedge \omega_{\alpha}-\sum_{r} \omega_{\alpha r} \wedge \sum_{t}\left(A_{r t}-\mu \delta_{r t}\right) \omega_{t}=0
$$

Setting $d \mu=\sum_{\imath} \mu_{\imath} \omega_{i}$, we can put

$$
\mu_{t} \omega_{\alpha}+\sum_{r} \omega_{\alpha r}\left(A_{r t}-\mu \delta_{r t}\right)=\sum_{s} K_{\alpha t s} \omega_{s}, \quad K_{\alpha t s}=K_{\alpha s t},
$$

by E. Cartan's lemma. Thus we have

$$
\sum_{r}\left(A_{r t}-\mu \delta_{r t}\right) \sum_{\alpha} \omega_{\alpha} \wedge \omega_{\alpha r}=\sum_{\alpha, s} K_{\alpha t s} \omega_{\alpha} \wedge \omega_{s}
$$


which implies

$$
\sum_{\alpha} \omega_{\alpha} \wedge \omega_{\alpha r}=0 \quad\left(\bmod \omega_{m+1}, \cdots, \omega_{n}\right)
$$

because

$$
\operatorname{det}\left(A_{r t}-\mu \delta_{r t}\right) \neq 0
$$

by the regularity of $\mu$. On the other hand, we have also

$$
\begin{aligned}
d \omega_{t} & =\sum_{\alpha} \omega_{\alpha} \wedge \omega_{\alpha t}+\sum_{r} \omega_{r} \wedge \omega_{r t} \\
& \equiv 0 \quad\left(\bmod \omega_{m+1}, \cdots, \omega_{n}\right)
\end{aligned}
$$

by (1.6). This shows that the system of Pfaff equations:

$$
\omega_{m+1}=\cdots=\omega_{n}=0,
$$

i. e. the distribution $E(\mu)$ is completely integrable.

Q.E.D.

Remark. We have proved Theorem A under the condition of regularity of the other principal curvatures (Theorem 2 in [3]).

We prove the following

THEOREM B. Let $M$ be a hypersurface immersed in an $(n+1)$-dimensional Riemannian manifold $\bar{M}$ of constant curvature $\bar{c}$. If the $3 r d$ fundamental form of $M$ vanishes, then $M$ is totally geodesic or umbilic, otherwise $M$ has just two principal curvatures $\lambda, \mu$ such that $\lambda \mu=-\bar{c}$.

Proof. Since we have $A_{\imath \jmath, k}=0$, we can choose special frames such that $A_{i i}=\lambda_{\imath}, A_{\imath j}=0(i \neq j)$ and $\lambda_{1}, \cdots, \lambda_{n}$ are all constant. If $\lambda_{1}=\lambda_{2}=\cdots=\lambda_{n}$, then $M$ is totally geodesic or umbilic. Otherwise, we have $\omega_{i j}=0$ for $\lambda_{i} \neq \lambda_{\text {, }}$. In fact, for such $i$ and $\jmath$, we have from (1.3)

$$
0=d A_{\imath j}-\sum_{k} \omega_{i k} A_{k j}-\sum_{k} \omega_{j k} A_{i k}=\left(\lambda_{i}-\lambda_{j}\right) \omega_{i \jmath},
$$

hence $\omega_{i j}=0$. Furthermore, from this relation we get

$$
\begin{aligned}
0 & =d \omega_{i j}=\sum_{k} \omega_{i k} \wedge \omega_{k \jmath}+\omega_{i(n+1)} \wedge \omega_{(n+1) \jmath}-\bar{c} \omega_{i} \wedge \omega_{\jmath} \\
& =-\left(\lambda_{2} \lambda_{\jmath}+\bar{c}\right) \omega_{i} \wedge \omega_{\jmath},
\end{aligned}
$$

i.e. $\quad \lambda_{2} \lambda_{j}+\bar{c}=0$, for $\lambda_{i} \neq \lambda_{\jmath}$.

This fact shows that the number of different principal curvatures is at most two.

Q.E.D.

Theorem B shows that if a hypersurface $M$ in $\bar{M}$ have more than two principal curvatures, its 3 rd fundamental form $\Psi$ does not vanish.

In the following, we consider also $\Psi$ as a symmetric tensor field of $M$. We denote the tangent space $M$ at $x \in M$ by $M_{x}$ and the inner product of $X, Y \in M_{x}$ by $\langle X, Y\rangle$. 


\section{§ 2. On the distributions for two regular principal curvature fields}

In this section, we shall consider $M$ in $\bar{M}$ as in Theorem A, whose 3rd fundamental form $\Psi$ does not vanish.

LEMMA 1. Let $\mu$ be a regular principal curvature field of $M$. For any tangent vectors $X, Y \in E(\mu, x)$, such that $\langle X, Y\rangle=0$ and any $Z \in M_{x}$, we have

$$
\Psi(X, Y, Z)=0 \text {. }
$$

Proof. Let us put $\operatorname{dim} E(\mu, x)=m$. We restrict $\omega_{i}, \omega_{i \jmath}, A_{\imath \jmath}$ on the submanifold of the frames $b=\left(x, e_{1}, \cdots, e_{n}, e_{n+1}\right)$ such that $e_{\alpha} \in E(\mu, x), \alpha=1,2, \cdots, m$. Then we have

$$
A_{\alpha \imath}=\mu \delta_{\alpha \imath}, \alpha=1,2, \cdots, m ; \imath=1,2, \cdots, n .
$$

Hence, by (1.3) we get

$$
\begin{aligned}
\sum_{k=1}^{n} B_{\alpha i k} \omega_{k} & =\delta_{\alpha i} d \mu-\sum_{k=1}^{n} \omega_{\alpha k} A_{k i}-\sum_{k=1}^{n} \omega_{i k} A_{\alpha k} \\
& =\delta_{\alpha i} d \mu-\mu \sum_{\gamma=1}^{m} \delta_{\gamma i} \omega_{\alpha \gamma}-\sum_{t=m+1}^{n} \omega_{\alpha t} A_{t i}+\mu \omega_{\alpha \imath} .
\end{aligned}
$$

Especially, we have

$$
\sum_{k=1}^{n} B_{\alpha_{\xi} k} \omega_{k}=\delta_{\alpha \beta} d \mu, \quad \alpha, \beta=1,2, \cdots, m,
$$

which imply easily this lemma.

Q.E.D.

LEMMA 2. If $\operatorname{dim} E(\mu)=\operatorname{dim} E(\mu, x) \geqq 2$, then

$$
\Psi(X, Y, Z)=0, \quad X, Y, Z \in E(\mu, x) .
$$

Proof. On the submanifold of $B$ used in the proof of Lemma 1, we have from (2.2)

$$
d \mu=\sum_{k=1}^{n} B_{\alpha \alpha^{k} \omega_{k}}=B_{\alpha \alpha \alpha \alpha} \omega_{\alpha}+\sum_{r=m+1}^{n} B_{\alpha \alpha r} \omega_{r}
$$

for a fixed $\alpha \leqq m$. Hence, putting $d \mu=\sum_{k=1}^{n} \mu{ }_{k} \omega_{k}$, we have

$$
\mu,{ }_{k}=B_{\alpha \alpha k}, \quad \alpha=1,2, \cdots, m ; k=1,2, \cdots, n
$$

and then, using the assumption $m \geqq 2$, we obtain

$$
\mu_{1}=\cdots=\mu_{,_{m}}=0 .
$$

Hence we have $B_{\alpha_{\beta} \gamma}=0$, which imply the lemma.

Q.E.D.

Since any integral submanifold of the distribution $E(\mu)$ is a solution of the system of Pfaff equations 


$$
\omega_{m+1}=\cdots=\omega_{n}=0,
$$

we have easily the following

COROLlary. If $\operatorname{dim} E(\mu) \geqq 2, \mu$ is constant on any integral submanifold of the distribution $E(\mu)$.

LEMMA 3. Let $\mu_{1}$ and $\mu_{2}$ be two regular principal curvature fields of $M$. Let $E\left(\mu_{1}\right)+E\left(\mu_{2}\right)$ be the distribution of the tangent subspace $E\left(\mu_{1}, x\right)+E\left(\mu_{2}, x\right)$, $x \in M$. Then, $E\left(\mu_{1}\right)+E\left(\mu_{2}\right)$ is completely integrable, if and only if for any $X \in$ $E\left(\mu_{1}, x\right), Y \in E\left(\mu_{2}, x\right)$ and any $Z \perp E\left(\mu_{1}, x\right)+E\left(\mu_{2}, x\right)$ we have $\Psi(X, Y, Z)=0$.

Proof. Let us put $\operatorname{dim} E\left(\mu_{1}, x\right)=m_{1}$ and $\operatorname{dim} E\left(\mu_{2}, x\right)=m_{2}$. We restrict $\omega_{1}, \omega_{i \jmath}, A_{\imath}$, on the submanifold of the frames $b=\left(x, e_{1}, \cdots, e_{n}, e_{n+1}\right)$ such that $e_{\alpha_{1}}$ $\in E\left(\mu_{1}, x\right), \alpha_{1}=1, \cdots, m_{1}$, and $e_{\alpha_{2}} \in E\left(\mu_{2}, x\right), \alpha_{2}=m_{1}+1, \cdots, m_{1}+m_{2}$. Then, we have

$$
\begin{gathered}
A_{\alpha_{1} 2}=\mu_{1} \delta_{\alpha_{1}} \text { and } A_{\alpha_{2} 2}=\mu_{2} \delta_{\alpha_{2} \imath} \\
\alpha_{1}=1, \cdots, m_{1} ; \alpha_{2}=m_{1}+1, \cdots, m_{1}+m_{2}, \imath=1,2, \cdots, n .
\end{gathered}
$$

From (2.1) and (2.3), we have for $s>m_{1}+m_{2}=m$

$$
\begin{aligned}
\mu_{1, s} \omega_{\alpha_{1}}+\sum_{\alpha_{2}} B_{\alpha_{1} \alpha_{2} s} \omega_{\alpha_{2}}+\sum_{t>m} B_{\alpha_{1} t s} \omega_{t} \\
=\left(\mu_{1}-A_{s s}\right) \omega_{\alpha_{1} s}-\sum_{t>m, t \neq s} \omega_{\alpha_{1} t} A_{t s},
\end{aligned}
$$

hence

$$
\begin{aligned}
\sum_{\alpha_{1}, \alpha_{2}} B_{\alpha_{1} \alpha_{2} s} \omega_{\alpha_{1}} \wedge \omega_{\alpha_{2}}+\sum_{\alpha_{1}} \sum_{t>m} B_{\alpha_{1} t s} \omega_{\alpha_{1}} \wedge \omega_{t} \\
=\left(\mu_{1}-A_{s s}\right) \sum_{\alpha_{1}} \omega_{\alpha_{1}} \wedge \omega_{\alpha_{1} s}-\sum_{t>m, t \neq s} A_{t s} \omega_{\alpha_{1}} \wedge \omega_{\alpha_{1} t} .
\end{aligned}
$$

Analogously, we get

$$
\begin{aligned}
\sum_{\alpha_{1}, \alpha_{2}} B_{\alpha_{1} \alpha_{2} s} \omega_{\alpha_{2}} \wedge \omega_{\alpha_{1}}+\sum_{\alpha_{2}} \sum_{t>m} B_{\alpha_{2} t s} \omega_{\alpha_{2}} \wedge \omega_{t} \\
=\left(\mu_{2}-A_{s s}\right) \sum_{\alpha_{2}} \omega_{\alpha_{2}} \wedge \omega_{\alpha_{2} s}-\sum_{t>m, t \neq s} A_{t s} \omega_{\alpha_{2}} \wedge \omega_{\alpha_{2} t} .
\end{aligned}
$$

Here, we restrict locally the submanifold of the above frames to the one such that $A_{t s}=\delta_{t s} \lambda_{t}, t, s>m$. Then, from the regularity of $\mu_{1}$ and $\mu_{2}$, we have $\mu_{1} \neq \lambda_{s}$, $\mu_{2} \neq \lambda_{s}, s>m$. Hence the above equalities turn out

$$
\begin{aligned}
& \sum_{\alpha_{1}} \omega_{\alpha_{1}} \wedge \omega_{\alpha_{1} s}=\frac{1}{\mu_{1}-\lambda_{s}}\left\{\sum_{\alpha_{1}, \alpha_{2}} B_{\alpha_{1} \alpha_{2} s} \omega_{\alpha_{1}} \wedge \omega_{a_{2}}+\sum_{\alpha_{1}} \sum_{t>m} B_{\alpha_{1} t_{s}} \omega_{\alpha_{1}} \wedge \omega_{t}\right\}, \\
& \sum_{\alpha_{2}} \omega_{\alpha_{2}} \wedge \omega_{\alpha_{2} s}=\frac{1}{\mu_{2}-\lambda_{s}}\left\{-\sum_{\alpha_{1}, \alpha_{2}} B_{\alpha_{1} \alpha_{2} s} \omega_{\alpha_{1}} \wedge \omega_{\alpha_{2}}+\sum_{\alpha_{2}} \sum_{t>m} B_{\alpha_{2} t s} \omega_{\alpha_{2}} \wedge \omega_{t}\right\} .
\end{aligned}
$$

Making use of these equalities, we get

$$
d \omega_{s}=\sum_{\alpha_{1}} \omega_{\sigma_{1}} \wedge \omega_{\alpha_{1} s}+\sum_{\alpha_{2}} \omega_{\alpha_{2}} \wedge \omega_{\alpha_{2} s}+\sum_{t>m} \omega_{t} \wedge \omega_{t s}
$$




$$
\begin{aligned}
= & -\frac{\mu_{1}-\mu_{2}}{\left(\mu_{1}-\lambda_{s}\right)\left(\mu_{2}-\lambda_{s}\right)} \sum_{\alpha_{1}, \alpha_{2}} B_{\alpha_{1} \alpha_{2} s} \omega_{\alpha 1} \wedge \omega_{\alpha 2} \\
& +\frac{1}{\mu_{1}-\lambda_{s}} \sum_{\alpha_{1}} \sum_{t>m} B_{\alpha_{1} t s} \omega_{\alpha_{1}} \wedge \omega_{t}+\frac{1}{\mu_{2}-\lambda_{s}} \sum_{\alpha_{2}} \sum_{t>m} B_{\alpha_{2} t s} \omega_{\alpha_{2}} \wedge \omega_{t} \\
& +\sum_{t>m} \omega_{t} \wedge \omega_{t s} .
\end{aligned}
$$

Since $E\left(\mu_{1}\right)+E\left(\mu_{2}\right)$ is given by $\omega_{s}=0, s>m$, it is completely integrable, if and only if

$$
\begin{gathered}
B_{\alpha_{1} \alpha_{2} s}=0, \\
\alpha_{1}=1, \cdots, m_{1} ; \alpha_{2}=m_{1}+1, \cdots, m_{1}+m_{2} ; s=m_{1}+m_{2}+1, \cdots, n,
\end{gathered}
$$

by means of (2.5). (2.6) is equivalent to the condition in the statement of this lemma.

Q.E.D.

From Lemma 3, we obtain easily the following

THEOREM 1. Let $M$ be a hypersurface immersed in a Riemannian manifold $\bar{M}$ of constant curvature and $\Psi$ its $3 r d$ fundamental form. If $M$ has just three regular principal curvature fields $\mu_{1}, \mu_{2}, \mu_{3}$ such that $E\left(\mu_{1}\right)+E\left(\mu_{2}\right)+E\left(\mu_{3}\right)=T M$, then the distributions $E\left(\mu_{2}\right)+E\left(\mu_{3}\right), E\left(\mu_{3}\right)+E\left(\mu_{1}\right)$ and $E\left(\mu_{1}\right)+E\left(\mu_{2}\right)$ are simultaneously completely integrable if and only if for any $X_{i} \in E\left(\mu_{2}, x\right), x \in M, i=1,2,3$,

$$
\Psi\left(X_{1}, X_{2}, X_{3}\right)=0 \text {. }
$$
becomes

In the case of integrable in Theorem 1, by (2.2) and (2.3) the equation (2.5)

$$
\begin{aligned}
d \omega_{\alpha_{3}}= & -\frac{\mu_{1}-\mu_{2}}{\left(\mu_{1}-\mu_{3}\right)\left(\mu_{2}-\mu_{3}\right)} \sum_{\alpha_{1}, \alpha_{2}} B_{\alpha_{1} o_{2} a_{3}} \omega_{\alpha_{1}} \wedge \omega_{\alpha_{2}} \\
& +\frac{1}{\mu_{1}-\mu_{3}} \sum_{\alpha_{1}} \mu_{3, \alpha_{1}} \omega_{\alpha_{1}} \wedge \omega_{\alpha_{3}}+\frac{1}{\mu_{2}-\mu_{3}} \sum_{\alpha_{2}} \mu_{3, a_{2}} \omega_{\alpha_{2}} \wedge \omega_{\alpha_{3}} \\
& +\sum_{\beta_{3}} \omega_{\beta_{3}} \wedge \omega_{\beta_{3} \alpha_{3}},
\end{aligned}
$$

where $e_{\alpha_{1}} \in E\left(\mu_{1}, x\right), \alpha_{1}=1, \cdots, m_{1} ; e_{\alpha_{2}} \in E\left(\mu_{2}, x\right), \alpha_{2}=m_{1}+1, \cdots, m_{1}+m_{2} ; e_{\alpha_{3}} \in E\left(\mu_{3}, x\right)$, $\alpha_{3}=m_{1}+m_{2}+1, \cdots, n$.

\section{§3. Minimal hypersurfaces with three non-simple regular principal cur- vature fields}

Using the notation in $\S 2$, we shall investigate a minimal hypersurface $M$ in $\bar{M}$ as in Theorem 1 with

$$
\Psi\left(X_{1}, X_{2}, X_{3}\right)=0 \quad \text { for } \quad X_{i} \in E\left(\mu_{\imath}, x\right), x \in M, \imath=1,2,3
$$

and 


$$
\operatorname{dim} E\left(\mu_{i}, x\right)=m_{i} \geqq 2, \imath=1,2,3,
$$

in this section.

LEMMA 4. There exist a local coordinate system $u_{1}, \cdots, u_{n}$ and functions $f_{1}$ $=f_{1}\left(u_{\alpha_{1}}\right), f_{2}=f_{2}\left(u_{\alpha_{2}}\right), f_{3}=f_{3}\left(u_{\alpha_{3}}\right)$ such that

$$
\mu_{1}=\frac{1}{m_{1}}\left(f_{2}-f_{3}\right), \quad \mu_{2}=\frac{1}{m_{2}}\left(f_{3}-f_{1}\right), \quad \mu_{3}=\frac{1}{m_{3}}\left(f_{1}-f_{2}\right) .
$$

Proof. Since $M$ is minimal in $\bar{M}$, we have

$$
m_{1} \mu_{1}+m_{2} \mu_{2}+m_{3} \mu_{3}=0 \text {. }
$$

By (3.1), we have

$$
B_{\alpha_{1} \alpha_{2} a_{3}}=0, \quad \alpha_{\imath} \in I\left(\mu_{\imath}\right)
$$

where

$$
I\left(\mu_{1}\right)=\left\{1,2, \cdots, m_{1}\right\}, \quad I\left(\mu_{2}\right)=\left\{m_{1}+1, \cdots, m_{1}+m_{2}\right\}, \quad I\left(\mu_{3}\right)=\left\{m_{1}+m_{2}+1, \cdots, n\right\} .
$$

By (3.2), (2.4), (2.7) and (3.4), there exist a local coordinate system $u_{1}, \cdots, u_{n}$ such that $u_{\alpha i}=$ constant, $\alpha_{i} \in I\left(\mu_{i}\right)$, give an integral submanifold of the distribution $E\left(\mu_{j}\right)+E\left(\mu_{k}\right),\{\imath, \jmath, k\}=\{1,2,3\}$, and $\mu_{i}=\mu_{i}\left(u_{\alpha_{j}} ; u_{a_{k}}\right)$. Taking a fixed point of $M$ with local coordinates $\left(c_{1}, \cdots, c_{n}\right)$, from (3.3) we get easily the following equalities:

$$
\begin{aligned}
& m_{2} \mu_{2}\left(u_{\alpha_{1}} ; u_{\alpha_{3}}\right)+m_{3} \mu_{3}\left(u_{\alpha_{1}} ; u_{\alpha_{2}}\right)=m_{2} \mu_{2}\left(c_{\alpha_{1}} ; u_{\alpha_{3}}\right)+m_{3} \mu_{3}\left(c_{\alpha_{1}} ; u_{\alpha_{2}}\right), \\
& m_{3} \mu_{3}\left(u_{\alpha_{1}} ; u_{\alpha_{2}}\right)+m_{1} \mu_{1}\left(u_{\alpha_{2}} ; u_{\alpha_{3}}\right)=m_{3} \mu_{3}\left(u_{\alpha_{1}} ; c_{\alpha_{2}}\right)+m_{1} \mu_{1}\left(c_{\alpha_{2}} ; u_{\alpha_{3}}\right), \\
& m_{1} \mu_{1}\left(u_{\alpha_{2}} ; u_{\alpha_{3}}\right)+m_{2} \mu_{2}\left(u_{\alpha_{1}} ; u_{\alpha_{3}}\right)=m_{1} \mu_{1}\left(u_{\alpha_{2}} ; c_{\alpha_{3}}\right)+m_{2} \mu_{2}\left(u_{\alpha_{1}} ; c_{\alpha_{3}}\right),
\end{aligned}
$$

from which we obtain

$$
\begin{gathered}
\left\{m_{2} \mu_{2}\left(u_{\alpha_{1}} ; c_{\alpha_{3}}\right)+m_{3} \mu_{3}\left(u_{\alpha_{1}} ; c_{\alpha_{2}}\right)\right\}+\left\{m_{3} \mu_{3}\left(c_{\alpha_{1}} ; u_{\alpha_{2}}\right)+m_{1} \mu_{1}\left(u_{\alpha_{2}} ; c_{\alpha_{3}}\right)\right\} \\
+\left\{m_{1} \mu_{1}\left(c_{\alpha_{2}} ; u_{\alpha_{3}}\right)+m_{2} \mu_{2}\left(c_{\alpha_{1}} ; u_{\alpha_{3}}\right)\right\}=0 .
\end{gathered}
$$

Hence there exist constants $a_{1}, a_{2}, a_{3}$ such that

and

$$
\begin{aligned}
& m_{2} \mu_{2}\left(u_{\alpha_{1}} ; c_{\alpha_{3}}\right)+m_{3} \mu_{3}\left(u_{\alpha_{1}} ; c_{\alpha_{2}}\right)=a_{1}, \\
& m_{3} \mu_{3}\left(c_{\alpha_{1}} ; u_{\alpha_{2}}\right)+m_{1} \mu_{1}\left(u_{\alpha_{2}} ; c_{\alpha_{3}}\right)=a_{2}, \\
& m_{1} \mu_{1}\left(c_{\alpha_{2}} ; u_{\alpha_{3}}\right)+m_{2} \mu_{2}\left(c_{\alpha_{1}} ; u_{\alpha_{3}}\right)=a_{3}
\end{aligned}
$$

Now, setting

$$
a_{1}+a_{2}+a_{3}=0 \text {. }
$$

$$
\begin{aligned}
& f_{1}\left(u_{\alpha_{1}}\right)=m_{3} \mu_{3}\left(u_{\alpha_{1}} ; c_{\alpha_{2}}\right)+a_{3}, f_{2}\left(u_{\alpha_{2}}\right)=-m_{3} \mu_{3}\left(c_{\alpha_{1}} ; u_{\alpha_{2}}\right), \\
& f_{3}\left(u_{\alpha_{3}}\right)=m_{2} \mu_{2}\left(c_{\alpha_{1}} ; u_{\alpha_{3}}\right),
\end{aligned}
$$

we have easily from (3.5) and (3.6) 
and

$$
\begin{aligned}
m_{1} \mu_{1}\left(u_{\alpha_{2}} ; u_{\alpha_{3}}\right) & =f_{2}\left(u_{\alpha_{2}}\right)-f_{3}\left(u_{\alpha_{3}}\right), \\
m_{2} \mu_{2}\left(u_{\alpha_{1}} ; u_{\alpha_{3}}\right) & =-m_{3} \mu_{3}\left(u_{\alpha_{1}} ; c_{\alpha_{2}}\right)-m_{1} \mu_{1}\left(c_{\alpha_{2}} ; u_{\alpha_{3}}\right) \\
& =-f_{1}\left(u_{\alpha_{1}}\right)+a_{3}-m_{1} \mu_{1}\left(c_{\alpha_{2}} ; u_{\alpha_{3}}\right) \\
& =-f_{1}\left(u_{\alpha_{1}}\right)+m_{2} \mu_{2}\left(c_{\alpha_{1}} ; u_{\alpha_{3}}\right)=f_{3}\left(u_{\alpha_{3}}\right)-f_{1}\left(u_{\alpha_{1}}\right)
\end{aligned}
$$

$$
m_{3} \mu_{3}\left(u_{\alpha_{1}} ; u_{\alpha_{2}}\right)=f_{1}\left(u_{\alpha_{1}}\right)-f_{2}\left(u_{\alpha_{2}}\right) \text {. }
$$

By Lemma 4, in the present case we obtain easily

$$
\mu_{2}-\mu_{3}=\frac{F-n f_{1}}{m_{2} m_{3}}, \quad \mu_{3}-\mu_{1}=\frac{F-n f_{2}}{m_{3} m_{1}}, \quad \mu_{1}-\mu_{2}=\frac{F-n f_{3}}{m_{1} m_{2}},
$$

and from (2.7)

$$
F=m_{1} f_{1}+m_{2} f_{2}+m_{3} f_{3}
$$

$$
\left\{\begin{array}{l}
d \omega_{\alpha_{1}}=-\left(\frac{m_{2} d f_{2}}{F-n f_{3}}+\frac{m_{3} d f_{3}}{F-n f_{2}}\right) \wedge \omega_{\alpha_{1}}+\sum_{\beta_{1}} \omega_{\beta_{1}} \wedge \omega_{\beta_{1} \alpha_{1}}, \\
d \omega_{\alpha_{2}}=-\left(\frac{m_{3} d f_{3}}{F-n f_{1}}+\frac{m_{1} d f_{1}}{F-n f_{3}}\right) \wedge \omega_{\alpha_{2}}+\sum_{\beta_{2}} \omega_{\beta_{2}} \wedge \omega_{\beta_{2} \alpha_{2}}, \\
d \omega_{\alpha_{3}}=-\left(\frac{m_{1} d f_{1}}{F-n f_{2}}+\frac{m_{2} d f_{2}}{F-n f_{1}}\right) \wedge \omega_{\alpha_{3}}+\sum_{\beta_{3}} \omega_{\beta_{3}} \wedge \omega_{\beta_{3} \alpha_{3}} .
\end{array}\right.
$$

Next, from (2.1) and (2.3) we obtain

hence

$$
\begin{aligned}
\left(\mu_{2}-\mu_{3}\right) \omega_{\alpha_{2} \alpha_{3}} & =\sum_{k=1}^{n} B_{\alpha_{2} \alpha_{3} k} \omega_{k}=B_{\alpha_{2} \alpha_{3} \alpha_{2}} \omega_{\alpha_{2}}+B_{\alpha_{2} \alpha_{3} \alpha_{3}} \omega_{\alpha_{3}} \\
& =\mu_{2, \alpha_{3}} \omega_{\alpha_{2}}+\mu_{3, \alpha_{2}} \omega_{\alpha_{3}}
\end{aligned}
$$

$$
\left\{\begin{array}{l}
\omega_{\alpha_{2} \alpha_{3}}=\frac{1}{F-n f_{1}}\left(m_{3} f_{3, \alpha_{3}} \omega_{\alpha_{2}}-m_{2} f_{2, \alpha_{2}} \omega_{\alpha_{3}}\right), \\
\omega_{\alpha_{3} \alpha_{1}}=\frac{1}{F-n f_{2}}\left(m_{1} f_{1, \alpha_{1}} \omega_{\alpha_{3}}-m_{3} f_{3, \alpha_{3}} \omega_{\alpha_{1}}\right), \\
\omega_{\alpha_{1} \alpha_{2}}=\frac{1}{F-n f_{3}}\left(m_{2} f_{2, \alpha_{2}} \omega_{\alpha_{1}}-m_{1} f_{1, \alpha_{1}} \omega_{\alpha_{2}}\right) .
\end{array}\right.
$$

Using these, we get

$$
\begin{aligned}
d \omega_{\alpha_{1} \beta_{1}}= & \sum_{k=1}^{n} \omega_{\alpha_{1} k} \wedge \omega_{k \beta_{1}}-\left(\mu_{1}^{2}+\bar{c}\right) \omega_{\alpha_{1}} \wedge \omega_{\beta_{1}} \\
= & \sum_{\gamma_{1}} \omega_{\alpha_{1} \gamma_{1}} \wedge \omega_{\gamma_{1} \beta_{1}}-\left(\mu_{1}^{2}+\bar{c}\right) \omega_{\alpha_{1}} \wedge \omega_{\beta_{1}} \\
& -\frac{1}{\left(F-n f_{3}\right)^{2}} \sum_{\gamma_{2}}\left(m_{2} f_{2, \gamma_{2}} \omega_{\alpha_{1}}-m_{1} f_{1, \alpha_{1}} \omega_{\gamma_{2}}\right) \wedge\left(m_{2} f_{2, \gamma_{2}} \omega_{\beta_{1}}-m_{1} f_{1, \beta_{1}} \omega_{\gamma_{2}}\right)
\end{aligned}
$$




$$
\begin{gathered}
-\frac{1}{\left(F-n f_{2}\right)^{2}} \sum_{\gamma 3}\left(m_{1} f_{1, \alpha_{1}} \omega_{\gamma_{3}}-m_{3} f_{3, \gamma_{3}} \omega_{\alpha_{1}}\right) \wedge\left(m_{1} f_{1, \beta_{1}} \omega_{\gamma_{3}}-m_{3} f_{3, \gamma_{3}} \omega_{\beta_{1}}\right) \\
=\sum_{\gamma 1} \omega_{\alpha_{1} \gamma_{1}} \wedge \omega_{\gamma_{1} \beta_{1}}-\left\{\frac{m_{2}^{2}}{\left(F-n f_{3}\right)^{2}}\left|\nabla f_{2}\right|^{2}+\frac{m_{3}^{2}}{\left(F-n f_{2}\right)^{2}}\left|\nabla f_{3}\right|^{2}+\mu_{1}^{2}+\bar{c}\right\} \omega_{\alpha_{1}} \wedge \omega_{\beta_{1}} \\
+\frac{m_{1} m_{2}}{\left(F-n f_{3}\right)^{2}}\left(\omega_{\alpha_{1}} \wedge f_{1, \beta_{1}} d f_{2}+f_{1, \alpha_{1}} d f_{2} \wedge \omega_{\beta_{1}}\right) \\
+\frac{m_{1} m_{3}}{\left(F-n f_{2}\right)^{2}}\left(\omega_{\alpha_{1}} \wedge f_{1, \beta_{1}} d f_{3}+f_{1, \alpha_{1}} d f_{3} \wedge \omega_{\beta_{1}}\right),
\end{gathered}
$$

i. e.

$$
\begin{aligned}
& d \omega_{\alpha_{1} \beta_{1}}-\sum_{\gamma_{1}} \omega_{\alpha_{1} \gamma_{1}} \wedge \omega_{\gamma_{1} \beta_{1}} \\
& =-\left[\frac{1}{\left(F-n f_{2}\right)^{2}\left(F-n f_{3}\right)^{2}}\left\{m_{2}^{2}\left(F-n f_{2}\right)^{2}\left|\nabla f_{2}\right|^{2}+m_{3}^{2}\left(F-n f_{3}\right)^{2}\left|\nabla f_{3}\right|^{2}\right\}\right.
\end{aligned}
$$

$$
\begin{aligned}
& \left.+\mu_{1}^{2}+\bar{c}\right] \omega_{\alpha_{1}} \wedge \omega_{\beta_{1}}+\frac{m_{1}}{\left(F-n f_{2}\right)^{2}\left(F-n f_{3}\right)^{2}}\left(f_{1, \beta_{1}} \omega_{\alpha_{1}}\right. \\
& \left.-f_{1, \alpha_{1}} \omega_{\beta_{1}}\right) \wedge\left\{m_{2}\left(F-n f_{2}\right)^{2} d f_{2}+m_{3}\left(F-n f_{3}\right)^{2} d f_{3}\right\} .
\end{aligned}
$$

Analogously, we get the formulas for $d \omega_{\alpha_{2} \beta_{2}}$ and $d \omega_{\alpha_{3} \beta_{3}}$ by cyclic changes of the suffixes in (3.10). Making use of the above formulas we get the following theorem.

THEOREM 2. Let $M$ be a minimal hypersurface immersed in a Riemannian manifold $\bar{M}$ of constant curvature with three non-simple regular principal curvature fields $\mu_{1}, \mu_{2}$ and $\mu_{3}$ such that $E\left(\mu_{1}\right)+E\left(\mu_{2}\right)+E\left(\mu_{3}\right)=T(M)$ and $\Psi\left(X_{1}, X_{2}, X_{3}\right)$ $=0$ for $X_{i} \in E\left(\mu_{i}, x\right), \imath=1,2,3, x \in M$. Then the integral submanifolds of $E\left(\mu_{i}\right)$ are totally umbilic in $\bar{M}$.

Proof. The integral submanifolds of the distribution $E\left(\mu_{1}\right)$ are the solutions of the Pfaff equations:

$$
\omega_{\alpha_{2}}=\omega_{\alpha_{3}}=0, \alpha_{2} \in I\left(\mu_{2}\right), \alpha_{3} \in I\left(\mu_{3}\right) .
$$

Hence, along any integral submanifold $K^{m_{1}}$ of $E\left(\mu_{1}\right)$ we have from (3.9), (1.2) and Lemma 4 the following:

$$
\begin{array}{ll}
\omega_{\alpha_{1} \beta_{2}}=\frac{m_{2}}{F-n f_{3}} f_{2, \beta_{2}} \omega_{\alpha_{1}}, & \beta_{2} \in I\left(\mu_{2}\right), \\
\omega_{\alpha_{1} \beta_{3}}=\frac{m}{F-n f_{2}} f_{3, \beta_{3}} \omega_{\alpha_{1}}, & \beta_{3} \in I\left(\mu_{3}\right), \\
\omega_{\alpha_{1}(n+1)}=\frac{1}{m_{1}}\left(f_{2}-f_{3}\right) \omega_{\alpha_{1}} . &
\end{array}
$$


These equalities show that $K^{m_{1}}$ is totally umbilic in $\bar{M}$.

Q.E.D.

By means of (3.12) the mean curvature vector field $H\left(\mu_{1}\right)$ of $K^{m_{1}}$ is given by

$$
\begin{aligned}
H\left(\mu_{1}\right)= & \frac{m_{2}}{F-n f_{3}} \nabla f_{2}+\frac{m_{3}}{F-n f_{2}} \nabla f_{3}+\frac{1}{m_{1}}\left(f_{2}-f_{3}\right) e_{n+1} \\
= & \frac{1}{\left(F-n f_{2}\right)\left(F-n f_{3}\right)}\left\{m_{2}\left(F-n f_{2}\right) \nabla f_{2}+m_{3}\left(F-n f_{3}\right) \nabla f_{3}\right\} \\
& +\frac{1}{m_{1}}\left(f_{2}-f_{3}\right) e_{n+1} .
\end{aligned}
$$

We have also for $K^{m_{1}}$ the following formulas:

$$
\left\{\begin{array}{l}
d x=\sum_{\alpha_{1}} \omega_{\alpha_{1}} e_{\alpha_{1}} \\
D e_{\alpha_{1}}=\sum_{\beta_{1}} \omega_{\alpha_{1} \beta_{1}} e_{\beta_{1}}+H\left(\mu_{1}\right) \omega_{\alpha_{1}}, \\
D e_{\alpha_{2}}=-\left\langle H\left(\mu_{1}\right), e_{\alpha_{2}}\right\rangle d x+\sum_{\beta_{2}} \omega_{\alpha_{2} \beta_{2}} e_{\beta_{2}}, \\
D e_{\alpha_{3}}=-\left\langle H\left(\mu_{1}\right), e_{\alpha_{3}}\right\rangle d x+\sum_{\beta_{3}} \omega_{\alpha_{3} \beta_{3}} e_{\beta_{3}}, \\
D e_{n+1}=-\left\langle H\left(\mu_{1}\right), e_{n+1}\right\rangle d x
\end{array}\right.
$$

where $D$ denotes the covariant differential operator of $\bar{M}$.

COROLlary. $K^{m_{1}}$ is a submanifold with m-index 0 in $\bar{M}$.

Proof. For any normal vector $\xi$ of $K^{m_{1}}$

$$
\xi=\sum_{\alpha_{2}} \xi_{\alpha_{2}} e_{\alpha_{2}}+\sum_{\alpha_{3}} \xi_{\alpha_{3}} e_{\alpha_{3}}+\xi_{n+1} e_{n+1},
$$

the corresponding 2nd fundamental form $A_{\hat{\xi}}$ is given by

$$
A_{\hat{\xi}}=\left\{\frac{m_{2}}{F-n f_{3}} \sum_{\beta_{2}} f_{2, \beta_{2}} \xi_{\beta_{2}}+\frac{m_{3}}{F-n f_{2}} \sum_{\beta_{3}} f_{3, \beta_{3}} \xi_{\beta_{3}}+\frac{1}{m_{1}}\left(f_{2}-f_{3}\right) \xi_{n+1}\right\} I .
$$

Hence, Trace $A_{\xi}=0$ if and only if $A_{\hat{\xi}}=0$. Therefore, the minimal index of $K^{m_{1}}$ (= $\operatorname{dim}\left\{A_{\tilde{\xi}} \mid\right.$ Trace $\left.A_{\hat{\xi}}=0\right\}$ ) must be zero (see $[1]$ ).

Q.E. D.

\section{§4. Certain minimal hypersurfaces in $\mathbf{S}^{n+1}$}

In this section, we shall investigate minimal hypersurfaces as in Theorem 2 in the case of $\bar{M}^{n+1}=S^{n+1}$ (unit $(n+1)$-sphere).

For any integrald submanifold $K^{m_{1}}$ of $E\left(\mu_{1}\right)$, (3.14) becomes 


$$
\left\{\begin{array}{l}
d x=\sum_{\alpha_{1}} \omega_{\alpha_{1}} e_{\alpha_{1}}, \\
d e_{\alpha_{1}}=\sum_{\beta_{1}} \omega_{\alpha_{1} \beta_{1}} e_{\beta_{1}}+\left(H\left(\mu_{1}\right)-x\right) \omega_{\alpha_{1}}, \\
d e_{\alpha_{2}}=-\left\langle H\left(\mu_{1}\right), e_{\alpha_{2}}\right\rangle d x+\sum_{\beta_{2}} \omega_{\alpha_{2} \beta_{2}} e_{\beta_{2}}, \\
d e_{\alpha_{3}}=-\left\langle H\left(\mu_{1}\right), e_{\alpha_{3}}\right\rangle d x+\sum_{\beta_{3}} \omega_{\alpha_{3} \beta_{3}} e_{\beta_{3}}, \\
d e_{n+1}=-\left\langle H\left(\mu_{1}\right), e_{n+1}\right\rangle d x,
\end{array}\right.
$$

where $d$ is the ordinary differential operator in $R^{n+2}\left(\supset S^{n+1}\right)$.

LEMMA 5. $K^{m_{1}}$ is an $m_{1}$-dimensional small sphere or an open subset of this sphere.

Proof. From (4.1), we get easily

$$
\begin{aligned}
0=d^{2} e_{\alpha_{1}}= & \sum_{\beta_{1}} d \omega_{\alpha_{1} \beta_{1}} e_{\beta_{1}}-\sum_{\beta_{1}} \omega_{\alpha_{1} \beta_{1}} \wedge\left\{\sum_{\gamma_{1}} \omega_{\beta_{1} \gamma_{1}} e_{\gamma_{1}}+\left(H\left(\mu_{1}\right)-x\right) \omega_{\beta_{1}}\right\} \\
& +\left(H\left(\mu_{1}\right)-x\right) d \omega_{\alpha_{1}}+d\left(H\left(\mu_{1}\right)-x\right) \wedge \omega_{\alpha_{1}} \\
= & \sum_{\beta_{1}}\left\{d \omega_{\alpha_{1} \beta_{1}}-\sum_{\gamma_{1}} \omega_{\alpha_{1} \gamma_{1}} \wedge \omega_{\gamma_{1} \beta_{1}}\right\} e_{\beta_{1}} \\
& +\left\{d \omega_{\alpha_{1}}-\sum_{\beta_{1}} \omega_{\alpha_{1} \beta_{1}} \wedge \omega_{\beta_{1}}\right\}\left(H\left(\mu_{1}\right)-x\right)+d\left(H\left(\mu_{1}\right)-x\right) \wedge \omega_{\alpha_{1}} .
\end{aligned}
$$

Hence, for any vector $Y$ orthogonal to $E\left(\mu_{1}, x\right)$ and $H\left(\mu_{1}\right)-x$, we have

$$
d\left\langle H\left(\mu_{1}\right)-x, Y>\wedge \omega_{\alpha_{1}}=0, \quad \alpha_{1}=1,2, \cdots, m_{1} .\right.
$$

Since $m_{1}=\operatorname{dim} E\left(\mu_{1}, x\right) \geqq 2$, this equalities imply

$$
\left\langle d\left(H\left(\mu_{1}\right)-x\right), Y\right\rangle=0, \quad \text { for } Y \perp E\left(\mu_{1}, x\right) \text { and } H\left(\mu_{1}\right)-x \text {. }
$$

By means of (4.1) and (4.2), we can easily see that the Euclidean $\left(m_{1}+1\right)$ vector $e_{1} \wedge \cdots \wedge e_{m_{1}} \wedge\left(H\left(\mu_{1}\right)-x\right)$ is parallel to a fixed one along $K^{m_{1}}$. Hence, there exists an $m_{1}+1$ dimensional Euclidean plane $E^{m_{1}+1} \supset K^{m_{1}}$.

Lemma 6. Let $M^{n}$ be as in Theorem 2 and $\bar{M}^{n+1}=S^{n+1}$. Then, at least two of the vector fields $H\left(\mu_{1}\right), H\left(\mu_{2}\right)$ and $H\left(\mu_{3}\right)$ do not vanish identically.

Proof. Assume that $H\left(\mu_{1}\right) \equiv H\left(\mu_{2}\right) \equiv 0$, Then, from (3.13) we obtain

$$
\nabla f_{1}=\nabla f_{2}=\nabla f_{3}=0, \quad f_{1}=f_{2}=f_{3} .
$$

Hence, $f_{1}, f_{2}$ and $f_{3}$ are the same constant, and so $\mu_{1}=\mu_{2}=\mu_{3}=0$ by Lemma 4 . This is a contradiction.

In the following of this section, we shall consider the case :

Q.E.D.

$$
H\left(\mu_{1}\right) \equiv 0 \quad \text { on } \quad M \text {. }
$$

From (3.13) we may put 


$$
f_{2}=f_{3}=a \quad \text { (constant). }
$$

By virtue of the proof of Lemma 5 , we see that the $\left(m_{1}+1\right)$-plane $E^{m_{1}+1}$ passes through the origin of $R^{n+2}$ in the present case, that is, the sphere $\supset K^{m_{1}}$ is an $m_{1}$-dimensional great sphere. We have from Lemma 4 , (3.9), (3.8) and (3.13) the following :

$$
\begin{gathered}
\mu_{1}=0, \quad \mu_{2}=-\frac{1}{m_{2}}\left(f_{1}-a\right), \quad \mu_{3}=\frac{1}{m_{3}}\left(f_{1}-a\right), \\
\left\{\begin{array}{l}
\omega_{\alpha_{2} \alpha_{3}}=0, \quad \omega_{\alpha_{3} \alpha_{1}}=\frac{1}{f_{1}-a} f_{1, \alpha_{1}} \omega_{\alpha_{3}}, \quad \omega_{\alpha_{1} \alpha_{2}}=-\frac{1}{f_{1}-a} f_{1, \alpha_{1}} \omega_{\alpha_{2}} \\
\omega_{\alpha_{1}(n+1)}=0, \quad \omega_{\alpha_{2}(n+1)}=-\frac{f_{1}-a}{m_{2}} \omega_{\alpha_{2}}, \quad \omega_{\alpha_{3}(n+1)}=\frac{f_{1}-a}{m_{3}} \omega_{\alpha_{3}}, \\
d \omega_{a_{2}}=-\frac{1}{f_{1}-a} d f_{1} \wedge \omega_{\alpha_{2}}+\sum_{\beta_{2}} \omega_{\alpha_{2} \beta_{2}} \wedge \omega_{\beta_{2}}, \\
d \omega_{\alpha_{3}}=-\frac{1}{f_{1}-a} d f_{1} \wedge \omega_{\alpha_{3}}+\sum_{\beta_{3}} \omega_{\alpha_{3} \beta_{3}} \wedge \omega_{\beta_{3}}
\end{array}\right.
\end{gathered}
$$

and

$$
H\left(\mu_{2}\right)=\frac{1}{f_{1}-a} \nabla f_{1}-\frac{f_{1}-a}{m_{2}} e_{n+1}, \quad H\left(\mu_{3}\right)=\frac{1}{f_{1}-a} \nabla f_{1}+\frac{f_{1}-a}{m_{3}} e_{n+1} .
$$

LEMma 7. Let $M^{n}$ be as in Theorem 2 and $\bar{M}^{n+1}=S^{n+1}$. If $H\left(\mu_{1}\right) \equiv 0$, then $f_{1}$ can not be constant.

Proof. Let us suppose that $H\left(\mu_{1}\right) \equiv 0$ and $f_{1}$ is a constant, then from Theorem 1 and (2.3) we obtain $\Psi \equiv 0$, which is impossible in the present case by Theorem $B$.

Q.E.D.

By this lemma, we see that $f_{1}$ is a non-constant function of $u_{1}, \cdots, u_{m_{1}}$. Now, we consider an integral submanifold $K^{m_{2}+m_{3}}$ of the distribution $E\left(\mu_{2}\right)+E\left(\mu_{3}\right)$. Along $K^{m_{2}+m_{3}}$, by (4.5) and (4.6) we have

$$
\left\{\begin{array}{l}
d x=\sum_{\alpha_{2}} \omega_{\alpha_{2}} e_{\alpha_{2}}+\sum_{\alpha_{3}} \omega_{\alpha_{3}} e_{\alpha_{3}}, \\
d e_{\alpha_{2}}=\sum_{\beta_{2}} \omega_{\alpha_{2} \beta_{2}} e_{\beta_{2}}+\left(H\left(\mu_{2}\right)-x\right) \omega_{\alpha_{2}}, \\
d e_{\alpha_{3}}=\sum_{\beta_{3}} \omega_{\alpha_{3} \beta_{3}} e_{\beta_{3}}+\left(H\left(\mu_{3}\right)-x\right) \omega_{\alpha_{3}}, \\
d e_{\alpha_{1}}=-\frac{1}{f_{1}-a} f_{1, \alpha_{1}} d x+\sum_{\beta_{1}} \omega_{\alpha_{1} \beta_{1}} e_{\beta_{1}}, \\
d e_{n+1}=\left(f_{1}-a\right)\left\{\frac{1}{m_{2}} \sum_{\alpha_{2}} \omega_{\alpha_{2}} e_{\alpha_{2}}-\frac{1}{m_{3}} \sum_{\alpha_{3}} \omega_{\alpha_{3}} e_{\alpha_{3}}\right\} .
\end{array}\right.
$$


LEMmA 8. Let $M^{n}$ be as in Theorem 2 and $\bar{M}^{n+1}=S^{n+1}$. If $H\left(\mu_{1}\right) \equiv 0$, then any integral submanifold $K^{m_{2}+m_{3}}$ of $E\left(\mu_{2}\right)+E\left(\mu_{3}\right)$ is locally contained in an $\left(m_{2}\right.$ $\left.+m_{3}+2\right)$-dimensional Euclidean plane in $R^{n+2}$ not containing the origin.

Proof. From (4.9), we get easily

$$
\begin{aligned}
0=d^{2} e_{\alpha_{2}}= & \sum_{\beta_{2}} d \omega_{\alpha_{2} \beta_{2}} e_{\beta_{2}}-\sum_{\beta_{2}} \omega_{a_{2} \beta_{2}} \wedge\left\{\sum_{\gamma_{2}} \omega_{\beta_{2} \gamma_{2}} e_{\gamma_{2}}+\left(H\left(\mu_{2}\right)-x\right) \omega_{\beta_{2}}\right\} \\
& +\left(H\left(\mu_{2}\right)-x\right) d \omega_{\alpha_{2}}+d\left(H\left(\mu_{2}\right)-x\right) \wedge \omega_{\alpha_{2}}
\end{aligned}
$$

i. e.

$$
\begin{aligned}
& \sum_{\beta_{2}}\left\{d \omega_{\alpha_{2} \beta_{2}}-\sum_{\gamma_{2}} \omega_{\alpha_{2} \gamma_{2}} \wedge \omega_{i_{2} \beta_{2}}\right\} e_{\beta_{2}} \\
& \quad+\left\{d \omega_{\alpha_{2}}-\sum_{\beta_{2}} \omega_{\alpha_{2} \beta_{2}} \wedge \omega_{\beta_{2}}\right\}\left\{\left(\frac{1}{f_{1}-a} \nabla f_{1}-x\right)-\frac{f_{1}-a}{m_{2}} e_{n+1}\right\} \\
& \quad+\left\{d\left(\frac{1}{f_{1}-a} \nabla f_{1}-x\right)-\frac{f_{1}-a}{m_{2}} d e_{n+1}\right\} \wedge \omega_{\alpha_{2}}=0,
\end{aligned}
$$

and analogously

$$
\begin{aligned}
& \sum_{\beta_{3}}\left\{d \omega_{\alpha_{3} \beta_{3}}-\sum_{\gamma_{3}} \omega_{\alpha_{3} \gamma_{3}} \wedge \omega_{\gamma_{3} \beta_{3}}\right\} e_{\beta_{3}} \\
& \quad+\left\{d \omega_{\alpha_{3}}-\sum_{\beta_{3}} \omega_{\alpha_{3} \beta_{3}} \wedge \omega_{\beta_{3}}\right\}\left\{\left(\frac{1}{f_{1}-a} \nabla f_{1}-x\right)+\frac{f_{1}-a}{m_{3}} e_{n+1}\right\} \\
& \quad+\left\{d\left(\frac{1}{f_{1}-a} \nabla f_{1}-x\right)+\frac{f_{1}-a}{m_{3}} d e_{n+1}\right\} \wedge \omega_{\alpha_{3}}=0 .
\end{aligned}
$$

Hence, for any vector $Y$ orthogonal to $E\left(\mu_{2}, x\right)+E\left(\mu_{3}, x\right), e_{n+1}$ and $\frac{1}{f_{1}-a} \nabla f_{1}-x$, we have

$$
d\left\langle\frac{1}{f_{1}-a} \nabla f_{1}-x, Y\right\rangle \wedge \omega_{\alpha_{2}}=0, \quad d\left\langle\frac{1}{f_{1}-a} \nabla f_{1}-x, Y\right\rangle \wedge \omega_{\alpha_{3}}=0,
$$

which imply

$$
\begin{aligned}
& \left\langle d\left(\frac{1}{f_{1}-a} \nabla f_{1}-x\right), Y\right\rangle=0 \\
& \quad \text { for } Y \perp E\left(\mu_{2}, x\right), E\left(\mu_{3}, x\right), e_{n+1}, \frac{1}{f_{1}-a} \nabla f_{1}-x .
\end{aligned}
$$

Now, by means of (4.9) and (4.10), we obtain easily the equality

$$
\begin{aligned}
& d\left\{\left(\frac{1}{f_{1}-a} \nabla f_{1}-x\right) \wedge e_{m_{1}+1} \wedge \cdots \wedge e_{n} \wedge e_{n+1}\right\} \\
= & d\left(\frac{1}{f_{1}-a} \nabla f_{1}-x\right) \wedge e_{m_{1}+1} \wedge \cdots \wedge e_{n} \wedge e_{n+1}
\end{aligned}
$$




$$
\begin{aligned}
= & \frac{1}{\left|\frac{1}{f_{1}-a} \nabla f_{1}\right|^{2}+1}\left\langle d\left(\frac{1}{f_{1}-a} \nabla f_{1}-x\right), \frac{1}{f_{1}-a} \nabla f_{1}-x\right\rangle \\
& \times\left(\frac{1}{f_{1}-a} \nabla f_{1}-x\right) \wedge e_{m_{1}+1} \wedge \cdots \wedge e_{n} \wedge e_{n+1}
\end{aligned}
$$

along $K^{m_{2}+m_{3}}$, which shows that the simple $\left(m_{2}+m_{3}+2\right)$-vector field

$$
\left(\frac{1}{f_{1}-a} \nabla f_{1}-x\right) \wedge e_{m_{1}+1} \wedge \cdots \wedge e_{n} \wedge e_{n+1}
$$

is parallel to a fixed $\left(m_{2}+m_{3}+2\right)$-dimensional direction in $R^{n+2}$. Therefore, there exists an $\left(m_{2}+m_{3}+2\right)$-dimensional Euclidean plane $\widetilde{E}^{m_{2}+m_{3}+2} \supset K^{m_{2}+m_{3}}$. Since $\nabla f_{1}$ $\neq 0, \widetilde{E}^{m_{2}+m_{3}+2}$ can not contain the origin of $R^{n+2}$.

Q.E.D.

Lemma 9. Let $M^{n}$ be as in Lemma 8 with $H\left(\mu_{1}\right) \equiv 0$. Then $K^{m_{2}+m_{3}}$ is a Riemannian product of an $m_{2}$-dimensional sphere and an $m_{3}$-dimensional sphere.

Proof. By Lemma $8, K^{m_{2}+m_{3}}$ is contained in the $\left(m_{2}+m_{3}+1\right)$-dimensional sphere $\widetilde{S}^{m_{2}+m_{3}+1}:=S^{n+1} \cap \widetilde{E}^{m 2+m_{3}+2}$ as a hypersurface with normal unit vector field $e_{n+1}$. In $\tilde{E}^{m_{2}+m_{3}+2}$ with the origin $\frac{1}{f_{1}-a} \nabla f_{1}$, the position vector of $x$ is given by

$$
y:=x-\frac{1}{f_{1}-a} \nabla f_{1} \text {. }
$$

Hence, in $\tilde{E}^{m_{2}+m_{3}+2},(4.9)$ can be written as

$$
\begin{aligned}
& d y=\sum_{\alpha_{2}} \omega_{\alpha_{2}} e_{\alpha_{2}}+\sum_{\alpha_{3}} \omega_{\alpha_{3}} e_{\alpha_{3}} \\
& d e_{\alpha_{2}}=\sum_{\beta_{2}} \omega_{\alpha_{2} \beta_{2}} e_{\beta_{2}}-\frac{f_{1}-a}{m_{2}} \omega_{\alpha_{2}} e_{n+1}-\omega_{\alpha_{2}} y, \\
& d e_{\alpha_{3}}=\sum_{\beta_{3}} \omega_{\alpha_{3} \beta_{3}} e_{\beta_{3}}+\frac{f_{1}-a}{m_{3}} \omega_{\alpha_{3}} e_{n+1}-\omega_{\alpha_{3}} y, \\
& d e_{n+1}=\left(f_{1}-a\right)\left\{\frac{1}{m_{2}} \sum_{\alpha_{2}} \omega_{\alpha_{2}} e_{\alpha_{2}}-\frac{1}{m_{3}} \sum_{\alpha_{3}} \omega_{\alpha_{3}} e_{\alpha_{3}}\right\} .
\end{aligned}
$$

These equalities show that $K^{m_{2}+m_{3}}$ is a minimal hypersurface in $\widetilde{S}^{m_{2}+m_{3}+1}$ with two principal curvatures $-\frac{f_{1}-a}{m_{2}}$ and $\frac{f_{1}-a}{m_{3}}$ of multiplicities $m_{2}$ and $m_{3}$ respectively. Hence, by Theorem 3 in [3], $K^{m_{2}+m_{3}}$ is locally a Riemannian product of two spheres of dimension $m_{2}$ and $m_{3}$ respectively.

Q.E.D.

Remark 1. The two spheres in Lemma 9 can be considered as integral submanifolds of the distribution $E\left(\mu_{2}\right)$ and $E\left(\mu_{3}\right)$ respectively. On the other hand, by means of the equalities in the proof Lemma 8 we obtain 
MINIMAL HYPERSURFACES WITH THREE PRINCIPAL

$$
\begin{aligned}
d \omega_{\alpha_{2} \beta_{2}}-\sum_{\gamma_{2}} \omega_{\alpha_{2} \gamma_{2}} \wedge \omega_{r_{2} \beta_{2}} & =\left\{\left\langle e_{\beta_{2}}, d y\right\rangle+\frac{f_{1}-a}{m_{2}}\left\langle e_{\beta_{2}}, d e_{n+1}\right\rangle\right\} \wedge \omega_{\alpha_{2}} \\
& =-\left\{1+\left(\frac{f_{1}-a}{m_{2}}\right)^{2}\right\} \omega_{\alpha_{2}} \wedge \omega_{\beta_{2}}, \\
d \omega_{\alpha_{3} \beta_{3}}-\sum_{\gamma_{3}} \omega_{\alpha_{3} \gamma_{3}} \wedge \omega_{\gamma_{3} \beta_{3}} & =\left\{\left\langle e_{\beta_{3}}, d y\right\rangle-\frac{f_{1}-a}{m_{3}}\left\langle e_{\beta_{3}}, d e_{n+1}\right\rangle\right\} \wedge \omega_{\alpha_{3}} \\
& =-\left\{1+\left(\frac{f_{1}-a}{m_{3}}\right)^{2}\right\} \omega_{\alpha_{3}} \wedge \omega_{\beta_{3}} .
\end{aligned}
$$

Hence, in $\widetilde{E}^{m_{2}+m_{3}+2}$ we have locally the product:

$$
K^{m_{2}+m_{3}} \cong \widetilde{S}^{m_{2}}\left(\left\{1+\left(\frac{f_{1}-a}{m_{2}}\right)^{2}\right\}^{-1 / 2}\right) \times \widetilde{S}^{m_{3}}\left(\left\{1+\left(\frac{f_{1}-a}{m_{3}}\right)^{2}\right\}^{-1 / 2}\right),
$$

where $\widetilde{S}^{m}(r)$ denotes the $m$-dimensional sphere of radius $r$.

Lemma 10. Let $M^{n}$ be as in Lemma 8 with $H\left(\mu_{1}\right) \equiv 0$. Then, $f_{1}$ satisfies:

$$
\left|\nabla f_{1}\right|^{2}=\frac{1}{m_{2} m_{3}}\left(f_{1}-a\right)^{4}-\left(f_{1}-a\right)^{2} .
$$

Proof. By means of (4.6) we have

$$
\begin{aligned}
0=d \omega_{\alpha_{2} \alpha_{3}} & =\sum_{j=1}^{n} \omega_{\alpha_{2} \jmath} \wedge \omega_{\jmath \alpha_{3}}+\omega_{\alpha_{2}(n+1)} \wedge \omega_{(n+1) \alpha_{3}}-\omega_{\alpha_{2}} \wedge \omega_{\alpha_{3}} \\
& =\sum_{\beta_{1}} \omega_{\alpha_{1} \beta_{1}} \wedge \omega_{\beta_{1} \alpha_{3}}-\left(\mu_{2} \mu_{3}+1\right) \omega_{\alpha_{2}} \wedge \omega_{\alpha_{3}} \\
& =-\left\{\frac{1}{\left(f_{1}-a\right)^{2}} \sum_{\beta_{1}}\left(f_{1, \beta_{1}}\right)^{2}-\frac{\left(f_{1}-a\right)^{2}}{m_{2} m_{3}}+1\right\} \omega_{\alpha_{2}} \wedge \omega_{\alpha_{3}},
\end{aligned}
$$

from which we get immediately (4.13).

Q.E. D.

Remark 2. Setting $\rho=\frac{1}{f_{1}-a}$, (4.13) becomes

$$
|\nabla \rho|^{2}+\rho^{2}=\frac{1}{m_{2} m_{3}} \quad \text { on } \quad M^{n} .
$$

Let $C$ be an orthogonal trajectory of the function $f_{1}$ parameterized with arclength $s$, then we get from (4.14)

$$
(d \rho / d s)^{2}+\rho^{2}=\frac{1}{m_{2} m_{3}},
$$

hence by integrating along $C$ we may put

$$
\rho=\frac{1}{\sqrt{m_{2} m_{3}}} \sin \left(s+c_{0}\right)
$$


where $c_{0}$ is a constant and $\sin \left(s+c_{0}\right) \neq 0$. This curve $C$ lies in an integral submanifold of $E\left(\mu_{1}\right)$, which is a great $m_{1}$-sphere of $S^{n+1}$ in the present case.

LEMma 11. Let $M$ be an n-dimensional Riemannian manifold and $u$ a nonconstant function such that $|\nabla u|^{2}$ is a non-zero function of $u$ only. Then, the integral curves of $\nabla u$ are geodesics of $M$ with certain parameters.

Proof. We choose local coordinates $x^{1}, \cdots, x^{n-1}, x^{n}$ such that $u=x^{n}$ and the metric of $M$ takes the form:

$$
d s^{2}=\sum_{\alpha, \beta=1}^{n} g_{\alpha \beta}(x) d x^{\alpha} d x^{\beta}+g_{n n}(x) d x^{n} d x^{n} .
$$

Then, we have $|\nabla u|^{2}=g^{n n}(x)$. From the assumption, we get

$$
\partial g^{n n} / \partial x^{\alpha}=-2 g^{n n} \Gamma_{n \alpha}^{n}=0,
$$

which imply $\partial g_{n n} / \partial x^{\alpha}=0, \alpha=1,2, \cdots, n-1$, where $\Gamma_{i k}^{\jmath}$ are the Christoffel symbols of the Riemannian connection of $M$.

On the other hand, the equations of a geodesic with respect to any parameter $t$ are

$$
\frac{\frac{d^{2} x^{1}}{d t^{2}}+\sum_{j, k} \Gamma_{j k}^{1} \frac{d x^{j}}{d t} \frac{d x^{k}}{d t}}{\frac{d x^{1}}{d t}}=\cdots \cdots=\frac{\frac{d^{2} x^{n}}{d t^{2}}+\sum_{j, k} \Gamma_{j k}^{n} \frac{d x^{j}}{d t} \frac{d x^{k}}{d t}}{\frac{d x^{n}}{d t}} .
$$

Now, for any curve $x^{\alpha}=$ constant, $\alpha=1,2, \cdots, n-1$, and $x^{n}=t$, we have

$$
\frac{d^{2} x^{\alpha}}{d t^{2}}+\sum_{j, k} \Gamma_{j k}^{\alpha} \frac{d x^{\jmath}}{d t} \frac{d x^{k}}{d t}=\Gamma_{n n}^{\alpha}=-\frac{1}{2} \sum_{\beta=1}^{n-1} g^{\alpha \beta} \frac{\partial g_{n n}}{\partial x^{\beta}}=0
$$

and

$$
\frac{d^{2} x^{n}}{d t^{2}}+\sum_{j, k} \Gamma_{j k}^{n} \frac{d x^{j}}{d t} \frac{d x^{k}}{d t}=\Gamma_{n n}^{n}=\frac{1}{2} g^{n n} \frac{\partial g_{n n}}{\partial x^{n}} .
$$

These equalities show that this curve is a geodesic of $M$.

Q.E. D.

Remark 3. In Lemma 11, if we suppose thal $|\nabla u|^{2}=F(u)$, then we obtain from the above computation $g_{n n}\left(x^{n}\right)=1 / F\left(x^{n}\right)$, Therefore, the arclength $s$ of the curve between $u=a$ and $u=b(a<b)$ is given by

$$
s=\int_{a}^{b} \frac{d u}{\sqrt{F(u)}} .
$$

Remark 4. By means of Lemma 11, Remark 2, Remark 3 and (4.14) the orthogonal trajectories of the function $f_{1}$ are all great circles of $S^{n+1}$. From these lemmas and remarks, we get the following theorem.

THEOREM 3. Let $M^{n}$ be as in Theorem 2 and $\bar{M}^{n+1}=S^{n+1}$ and $H\left(\mu_{1}\right) \equiv 0$. Then, we have the following:

(i) There exist a constant $a$ and a non-trivial function $f$ such that 


$$
\mu_{1}=0, \quad \mu_{2}=-\frac{1}{m_{2}}(f-a), \quad \mu_{3}=\frac{1}{m}(f-a) .
$$

(ii) Any integral submanifold of the distribution $E\left(\mu_{1}\right)$ is an $m_{1}$-dimensional great sphere of $S^{n+1}$ and any integral submanifolds of the distributions $E\left(\mu_{2}\right)$ and $E\left(\mu_{3}\right)$ are $m_{2}$ and $m_{3}$-dimensional small spheres of $S^{n+1}$ respectively.

(iii) Any integral submanifold of the distribution $E\left(\mu_{2}\right)+E\left(\mu_{3}\right)$ is a Riemannian product of an $m_{2}$-dimensional sphere and an $m_{2}$-dimensional sphere.

(iv) Any orthogonal trajectory $C$ of the level hypersurfaces of the function $f$ is an arc of a great circle of $\mathrm{S}^{n+1}$.

(v) As function of the arclength $s$ of $C, f$ can be written as

$$
f=a+\frac{\sqrt{m_{2} m_{3}}}{\sin \left(s+c_{0}\right)}
$$

where $c_{0}$ is a constant.

(v) of Theorem 3 implies immediately the following fact.

Corollary. Any n-dimensional complete Rremannian manrfold can not be isometrically immersed in $S^{n+1}$ as in Theorem 3.

\section{$\S 5$. An example of minimal hypersurfaces in $\mathbf{S}^{n+1}$ for Theorem 3}

In this section, we shall give an example of minimal hypersurfaces in $S^{n+1}$ with three non-simple regular principal curvatures $\mu_{1}, \mu_{2}$ and $\mu_{3}$ such that $H\left(\mu_{1}\right)$ $\equiv 0$, by making use of the facts obtained in $\S 4$.

Let $m_{1}, m_{2}$ and $m_{3}$ be any integers greater than 1 and put $n=m_{1}+m_{2}+m_{3}$. Let us consider as

$$
R^{n+2}=R^{m_{1}} \times R^{m_{2}+1} \times R^{m_{3}+1} .
$$

First of all, we take two hyperspheres in $R^{m_{2}+1}$ and $R^{m_{3}+1}$ as follows:

$$
\begin{array}{ll}
\tilde{S}_{0}^{m_{2}}\left(r_{2}\right) \subset R^{m_{2}+1}, & r_{2}=\left\{1+\left(\frac{b-a}{m_{2}}\right)^{2}\right\}^{-1 / 2}, \\
\tilde{S}_{0}^{m_{3}}\left(r_{3}\right) \subset R^{m_{3}+1}, & r_{3}=\left\{1+\left(\frac{b-a}{m_{3}}\right)^{2}\right\}^{-1 / 2}
\end{array}
$$

and put

$$
K_{0}^{m_{2}+m_{3}}=\tilde{S}_{0}^{m_{2}}\left(r_{2}\right) \times \widetilde{S}_{0}^{m_{3}}\left(r_{3}\right),
$$

which is contained in the $\left(m_{2}+m_{3}+1\right)$-dimensional sphere $\tilde{S}_{0}^{m_{2}+m_{3}+1}\left(r_{0}\right)$ in $R^{m_{2}+1}$ $\times R^{m_{3}+1}$, where

$$
r_{0}^{2}=r_{2}^{2}+r_{3}^{2}=\left\{2+\left(\frac{1}{m_{2}^{2}}+\frac{1}{m_{3}^{2}}\right)(b-a)^{2}\right\} /\left\{1+\left(\frac{b-a}{m_{2}}\right)^{2}\right\}\left\{1+\left(\frac{b-a}{m_{3}}\right)^{2}\right\} .
$$

From (5.2), we have 


$$
1-r_{0}^{2}=\left\{\frac{(b-a)^{4}}{\left(m_{2} m_{3}\right)^{2}}-1\right\} /\left\{1+\left(\frac{b-a}{m_{2}}\right)^{2}\right\}\left\{1+\left(\frac{b-a}{m_{3}}\right)^{2}\right\}:=r_{1}^{2}
$$

and so we suppose here the inequality:

$$
(b-a)^{2}>m_{2} m_{3} .
$$

Let $\eta$ be a normal unit vector field of $K_{0}^{m_{2}+m_{3}}$ in $\widetilde{S}_{0}^{m_{2}+m_{3}+1}\left(r_{0}\right)$.

Next, we take the hypersphere $\widetilde{S}_{0}^{m_{1}-1}\left(r_{1}\right) \subset R^{m_{1}}$, then we have

$$
\widetilde{S}_{0}^{m_{1}-1}\left(r_{1}\right) \times K_{0}^{m_{2}+m_{3}} \subset S^{n+1} .
$$

Let $x_{0}=\left(y_{0}, z_{0}\right)$ be any point of $\widetilde{S}_{0}^{m_{1}-1}\left(r_{1}\right) \times K_{0}^{m_{2}+m_{3}}$ and $E_{x_{0}}^{m_{1}}$ be the $m_{1}$-dimensional linear subspace of $R^{n+2}$ which is tangent to $S^{n+1}$ at $x_{0}$, orthogonal to $O \times T_{z_{0}}\left(K_{0}^{m_{2}+m_{3}}\right)$ and $O \times \eta\left(z_{0}\right)$. Let $S_{x_{0}}^{m_{1}}$ be the great $m_{1}$-sphere of $S^{n+1}$ which is the intersection of $S^{n+1}$ and $\left(m_{1}+1\right)$-dimensional linear subspace including $E_{x_{0}}^{m_{1}}$ and the origin of $R^{n+2}$. Then, we define a hypersurface of $S^{n+1}$ by

$$
M^{n}:=\cup\left\{S_{x_{0}}^{m_{1}} \mid x_{0} \in \widetilde{S}_{0}^{m_{1}-1}\left(r_{1}\right) \times K_{0}^{m_{2}+m_{3}}\right\} .
$$

In the rest of this section, we shall prove that this $M^{n}$ is a minimal hypersurface of $S^{n+1}$ as in Theorem 3 under an additional condition.

First, we define a tangent unit vector field $\xi$ of $M^{n}$ by the following way. At the point $x_{0}$ above, $\xi\left(x_{0}\right)$ be one of the tangent unit vectors to $S_{x_{0}}^{m_{1}}$ orthogonal to $T_{y_{0}}\left(S_{0}^{m_{1}-1}\left(r_{1}\right)\right) \times O$, since this makes sense by means of $\widetilde{S}_{0}^{m_{1}-1}\left(r_{1}\right) \times z_{0} \subset S_{x_{0}}^{m_{1}}$. Then, we extend the domain of definition of $\xi$ along the great circle $C_{x_{0}}$ of $S^{n+1}$ which passes through $x_{0}$ and has $\xi\left(x_{0}\right)$ as the tangent vector. Let $C_{x_{0}}$ be parameterized with arclength $s$ such that

$$
C_{x_{0}}: x=\gamma_{x_{0}}(s) \quad \text { with } \quad x_{0}=\gamma_{x_{0}}(0) .
$$

Then, considering ( $v$ ) in Theorem 3, we put

$$
c_{0}=\operatorname{Sin}^{-1} \frac{\sqrt{m_{2} m_{3}}}{b-a}
$$

and define a function $f$ on $M^{n}$ by

$$
f(x)=a+\frac{\sqrt{m_{2} m_{3}}}{\sin \left(s+c_{0}\right)},
$$

where $x=\gamma_{x_{0}}(s)$ and $0<s+c_{0}<\pi$.

Now, we compute the second fundamental form of $M^{n}$ in $S^{n+1}$. Setting

$$
x=(y, z), \quad z=(u, v), \quad y \in R^{m_{1}}, \quad u \in R^{m_{2}+1}, \quad v \in R^{m_{3}+1}
$$

and $z_{0}=\left(u_{0}, v_{0}\right)$, we have $\left|y_{0}\right|=r_{1},\left|u_{0}\right|=r_{2},\left|v_{0}\right|=r_{3}$. The normal unit vector $\eta\left(z_{0}\right)$ at $z_{0}$ of $K_{0}^{m_{2}+m_{3}}$ in $S_{0}^{m_{2}+m_{3}+1}\left(r_{0}\right)$ is given by

$$
\eta\left(z_{0}\right)=\left(\frac{r_{3}}{r_{0} r_{2}} u_{0},-\frac{r_{2}}{r_{0} r_{3}} v_{0}\right) .
$$


Analogously, we can obtain

$$
\xi\left(x_{0}\right)=\left(-\frac{r_{0}}{r_{1}} y_{0}, \frac{r_{1}}{r_{0}} u_{0}, \frac{r_{1}}{r_{0}} v_{0}\right)
$$

Hence, we get

$$
x=x_{0} \cos \theta+\xi\left(x_{0}\right) \sin \theta
$$

$$
=\left(\left(\cos \theta-\frac{r_{0}}{r_{1}} \sin \theta\right) y_{0},\left(\cos \theta+\frac{r_{1}}{r_{0}} \sin \theta\right) u_{0},\left(\cos \theta+\frac{r_{1}}{r_{0}} \sin \theta\right) v_{0}\right) .
$$

Next, we compute the normal unit vector $N\left(x_{0}\right)$ of $M^{n}$ in $S^{n+1}$ at $x_{0}$ by means of (5.10). We get easily

$$
N\left(x_{0}\right)=\left(0, \frac{r_{3}}{r_{0} r_{2}} u_{0},-\frac{r_{2}}{r_{0} r_{3}} v_{0}\right)=\left(0, \eta\left(z_{0}\right)\right) .
$$

From (5.11), we have at $x_{0}$

$$
d^{2} x:\left\{\begin{array}{l}
d^{2} y=d^{2} y_{0}-2 \frac{r_{0}}{r_{1}} d \theta d y_{0}-\left((d \theta)^{2}+\frac{r_{0}}{r_{1}} d^{2} \theta\right) y_{0}, \\
d^{2} u=d^{2} u_{0}+2 \frac{r_{1}}{r_{0}} d \theta d u_{0}-\left((d \theta)^{2}-\frac{r_{1}}{r_{0}} d^{2} \theta\right) u_{0}, \\
d^{2} v=d^{2} v_{0}+2 \frac{r_{1}}{r_{0}} d \theta d v_{0}-\left((d \theta)^{2}-\frac{r_{1}}{r_{0}} d^{2} \theta\right) v_{0}
\end{array}\right.
$$

and hence we get

$$
\begin{aligned}
\left\langle d^{2} x, N\left(x_{0}\right)\right\rangle= & \left\langle d^{2} y, 0\right\rangle+\frac{r_{3}}{r_{0} r_{2}}\left\langle d^{2} u, u_{0}\right\rangle-\frac{r_{2}}{r_{0} r_{3}}\left\langle d^{2} v, v_{0}\right\rangle \\
= & \frac{r_{3}}{r_{0} r_{2}}\left\{\left\langle d^{2} u_{0}, u_{0}\right\rangle-r_{2}^{2}\left((d \theta)^{2}-\frac{r_{1}}{r_{0}} d^{2} \theta\right)\right\} \\
& -\frac{r_{2}}{r_{0} r_{3}}\left\{\left\langle d^{2} v_{0}, v_{0}\right\rangle-r_{3}^{2}\left((d \theta)^{2}-\frac{r_{1}}{r_{0}} d^{2} \theta\right)\right\}
\end{aligned}
$$

i. e.

$$
\left\langle d^{2} x, N\left(x_{0}\right)\right\rangle=-\frac{r_{3}}{r_{0} r_{2}}\left\langle d u_{0}, d u_{0}\right\rangle+\frac{r_{2}}{r_{0} r_{3}}\left\langle d v_{0}, d v_{0}\right\rangle .
$$

On the other hand, we have also at $x_{0}$

$$
d x:\left\{\begin{array}{l}
d y=d y_{0}-\frac{r_{0}}{r_{1}} d \theta y_{0}, \\
d u=d u_{0}+\frac{r_{1}}{r_{0}} d \theta u_{0}, \\
d v=d v_{0}+\frac{r_{1}}{r_{0}} d \theta v_{0}
\end{array}\right.
$$


and hance we get

$$
d s^{2}=\left\langle d y_{0}, d y_{0}\right\rangle+d \theta^{2}+\left\langle d u_{0}, d u_{0}\right\rangle+\left\langle d v_{0}, d v_{0}\right\rangle .
$$

From (5.13) and (5.14), we see that $M^{n}$ have three principal curvatures $\mu_{1}=0, \mu_{2}$ $=-\frac{r_{3}}{r_{0} r_{2}}, \mu_{3}=\frac{r_{2}}{r_{0} r_{3}}$ of multiplicities $m_{1}, m_{2}, m_{3}$ respectively at $x_{0}$. From the argument above, especially the formula (5.11), we see that $\mu_{1}, \mu_{2}, \mu_{3}$ can be considered as regular fields and $\mu_{1} \equiv 0$, by replacing $r_{2}$ and $r_{3}$ with

$$
\bar{r}_{2}=\left(\cos \theta+\frac{r_{1}}{r_{0}} \sin \theta\right) r_{2}, \quad \bar{r}_{3}=\left(\cos \theta+\frac{r_{1}}{r_{0}} \sin \theta\right) r_{3}
$$

respectively. Then, we get easily

$$
H\left(\mu_{1}\right) \equiv 0 \text {. }
$$

Finally, we shall check the condition that $M^{n}$ is minimal in $S^{n+1}$. We have

$$
\begin{aligned}
m_{2} \mu_{2}+m_{3} \mu_{3} & =-\frac{m_{2} \bar{r}_{3}}{\bar{r}_{0} \bar{r}_{2}}+\frac{m_{3} \bar{r}_{2}}{\bar{r}_{0} \bar{r}_{3}} \\
& =\frac{\bar{r}_{2} \bar{r}_{3}}{\bar{r}_{0}}\left[-\frac{m_{2}}{\bar{r}_{2}^{2}}+\frac{m_{3}}{\bar{r}_{3}^{2}}\right]=\frac{r_{2} r_{3}}{\bar{r}_{0}}\left[-\frac{m_{2}}{r_{2}^{2}}+\frac{m_{3}}{r_{3}^{2}}\right] \\
& =\frac{r_{2} r_{3}}{\bar{r}_{0}}\left[-\left(m_{2}-m_{3}\right)+(b-a)^{2}\left(\frac{1}{m_{3}}-\frac{1}{m_{2}}\right)\right],
\end{aligned}
$$

that is

$$
m_{2} \mu_{2}+m_{3} \mu_{3}=\frac{\left(m_{2}-m_{3}\right) r_{2} r_{3}}{m_{2} m_{3} \bar{r}_{0}}\left\{(b-a)^{2}-m_{2} m_{3}\right\},
$$

where $\bar{r}_{0}=\sqrt{ } \bar{r}_{2}^{2}+\bar{r}_{3}^{2}$. Accordingly, by $(5,4), M^{n}$ is minimal if and only if

$$
m_{2}=m_{3} \text {. }
$$

Now, assuming (5.17), at a general point $x$ we have

$$
\mu_{2}=-\mu_{3}=-\frac{\bar{r}_{3}}{\bar{r}_{0} \bar{r}_{2}}=-\frac{1}{\sqrt{ } \overline{2} \bar{r}_{2}}=-\left\{\sqrt{2}\left(\cos \theta+\frac{r_{1}}{r_{0}} \sin \theta\right) r_{2}\right\}^{-1} .
$$

On the other hand, from (5.3), (5.7) and (5.17) we have

$$
\begin{gathered}
\frac{r_{1}}{r_{0}}=\frac{\sqrt{1-2 r_{2}^{2}}}{\sqrt{2} r_{2}}=\sqrt{\frac{1}{2}\left\{1+\left(\frac{b-a}{m_{2}}\right)^{2}\right\}-1}=\frac{1}{\sqrt{2}\left|\tan c_{0}\right|}, \\
r_{2}^{2}=\left\{1+\left(\frac{b-a}{m_{2}}\right)^{2}\right\}^{-1}=\frac{\sin ^{2} c_{0}}{1+\sin ^{2} c_{0}},
\end{gathered}
$$

and 


$$
\begin{aligned}
2\left(\cos \theta+\frac{r_{1}}{r_{0}} \sin \theta\right)^{2} r_{2}^{2} & =2\left(\cos \theta+\frac{\sin \theta}{\sqrt{\bar{L}}\left|\tan c_{0}\right|}\right)^{2} \times \frac{\sin ^{2} c_{0}}{1+\sin ^{2} c_{0}} \\
& =\frac{\left\{ \pm \cos \theta \cdot \sqrt{\zeta} \sin c_{0}+\sin \theta \cdot \cos c_{0}\right\}^{2}}{1+\sin ^{2} c_{0}}=\sin ^{2}\left(\theta+\theta_{0}\right),
\end{aligned}
$$

where

$$
\sin \theta_{0}= \pm \frac{\sqrt{L} \sin c_{0}}{\sqrt{1+\sin ^{2} c_{0}}}, \quad \cos \theta_{0}=\frac{\cos c_{0}}{\sqrt{1+\sin ^{2} c_{0}}},
$$

Hence, we may put

$$
\mu_{2}=-\mu_{3}=-\frac{1}{\sin \left(\theta+\theta_{0}\right)} .
$$

Thus, we obtain a conclusion as follows:

THEOREM 4. Let $M^{n}$ be a hypersurface constructed by (5.5) in $S^{n+1}$. Then, it is an example of minimal hypersurfaces as in Theorem 3, if and only if $m_{2}=m_{3}$.

Remark 5. Since we took $\widetilde{S}_{0}^{m_{1}-1} \times K_{0}^{m_{2}+m_{3}} \subset S^{n+1}$ as a base hypersurface of $M^{n}$ for our construction, the above argument does not entirely treat with this kind of minimal hypersurfaces in $S^{n+1}$.

\section{§ 6. The case in which $H\left(\mu_{2}\right) \neq 0, i=1,2,3$}

In this section, we shall investigate minimal hypersurfaces as in Theorem 2 with $H\left(\mu_{i}\right) \equiv \equiv, \imath=1,2,3$, in the case of $\bar{M}^{n+1}=S^{n+1}$.

By (3.13), $H\left(\mu_{1}\right) \equiv 0$ is equivalent to the condition

$$
f_{2}=f_{3}=a \text { constant } .
$$

LEMMA 12. Let $M^{n}$ be a minimal hypersurface in $S^{n+1}$ as in Theorem 2 with $H\left(\mu_{\imath}\right) \equiv 0, \imath=1,2,3$, then $f_{\imath}$ in Lemma 4 can not be constant for $\imath=1,2,3$.

Proof. Let $M^{n}$ be a hypersurface as in the statement. Let us suppose that $f_{\imath}=a_{\imath}, \imath=1,2,3$, where $a_{1}, a_{2}$ and $a_{3}$ are constants different from each others. Then, by (3.9) we have the equation

Hence we have from (1.3)

$$
\omega_{\alpha_{2} \alpha_{3}}=\omega_{\alpha_{3} \alpha_{1}}=\omega_{\alpha_{1} \alpha_{2}}=0 \text {. }
$$

$$
\begin{aligned}
D A_{\alpha_{\imath} \alpha_{j}} & =d A_{\alpha_{i} \alpha_{j}}-\sum_{k=1}^{n} \omega_{\alpha_{i} k} A_{k \alpha_{j}}-\sum_{k=1}^{n} \omega_{\alpha_{j} k} A_{\alpha_{i} k} \\
& =-\mu_{j} \omega_{\alpha_{i} \alpha_{j}}-\mu_{i} \omega_{\alpha_{j} \alpha_{\imath}}=\left(\mu_{\imath}-\mu_{\jmath}\right) \omega_{\alpha_{i} \alpha_{j}}=0,
\end{aligned}
$$

for $\imath, \jmath=1,2,3$, which imply $\Psi \equiv 0$. This contradicts to Theorem B. Q.E.D.

By means of Lemma 12, we may consider the case:

$$
f_{1}=f \text { is a non-constant function, } f_{2}=a_{2}, f_{3}=a_{3}, \quad a_{2} \neq a_{3},
$$


where $a_{2}$ and $a_{3}$ are constants, as the simplest one in the present situation. In the following, we shall investigate this case.

Under the condition (6.1), we have from Lemma 4 and (3.13)

$$
\begin{gathered}
\mu_{1}=\frac{a_{2}-a_{3}}{m_{1}}, \quad \mu_{2}=\frac{a_{3}-f}{m_{2}}, \quad \mu_{3}=\frac{f-a_{2}}{\cdot m_{3}}, \\
\left\{\begin{array}{l}
H\left(\mu_{1}\right)=\mu_{1} e_{n+1}, \\
H\left(\mu_{2}\right)=\frac{m_{1}}{F-n a_{3}} \nabla f+\mu_{2} e_{n+1}, \quad H\left(\mu_{3}\right)=\frac{m_{1}}{F-n a_{2}} \nabla f+\mu_{3} e_{n+1}
\end{array}\right.
\end{gathered}
$$

where

$$
F=m_{1} f+m_{2} a_{2}+m_{3} a_{3}, \quad n=m_{1}+m_{2}+m_{3} .
$$

From (3.8) and (3.9) we have

$$
\left\{\begin{array}{l}
d \omega_{\alpha_{1}}=\sum_{\beta_{1}} \omega_{\beta_{1}} \wedge \omega_{\beta_{1} \alpha_{1}}, \\
d \omega_{\alpha_{2}}=-\frac{m_{1}}{F-n a_{3}} d f \wedge \omega_{\alpha_{2}}+\sum_{\beta_{2}} \omega_{\beta_{2}} \wedge \omega_{\beta_{2} \alpha_{2}}, \\
d \omega_{\alpha_{3}}=-\frac{m_{1}}{F-n a_{2}} d f \wedge \omega_{\alpha_{3}}+\sum_{\beta_{3}} \omega_{\beta_{3}} \wedge \omega_{\beta_{3} \alpha_{3}}
\end{array}\right.
$$

and

$$
\omega_{\alpha_{2} \alpha_{3}}=0, \omega_{\alpha_{3} \alpha_{1}}=\frac{m_{1}}{F-n a_{2}} f_{,_{1}} \omega_{\alpha_{3}}, \quad \omega_{\alpha_{1} \alpha_{2}}=-\frac{m_{1}}{F-n a_{3}} f_{,_{\alpha_{1}}} \omega_{\alpha_{2}} .
$$

Now, by means of (6.3) and (6.6), we have the following equalities along $M^{n}$ :

$$
\left\{\begin{aligned}
d x= & \sum_{\alpha_{1}} \omega_{\alpha_{1}} e_{\alpha_{1}}+\sum_{\alpha_{2}} \omega_{\alpha_{2}} e_{\alpha_{2}}+\sum_{\alpha_{3}} \omega_{\alpha_{3}} e_{\alpha_{3}}, \\
d e_{\alpha_{1}}= & \sum_{\beta_{1}} \omega_{\alpha_{1} \beta_{1}} e_{\beta_{1}}-m_{1} f_{\alpha_{1}}\left\{\frac{1}{F-n a_{3}} \sum_{\beta_{2}} \omega_{\beta_{2} e_{2}} e_{\beta_{2}}+\frac{1}{F-n a_{2}} \sum_{\beta_{3}} \omega_{\beta_{3}} e_{\beta_{3}}\right\} \\
& \quad+\left(\mu_{1} e_{n+1}-x\right) \omega_{\alpha_{1}}, \\
d e_{\alpha_{2}}= & \sum_{\beta_{2}} \omega_{\alpha_{2} \beta_{2}} e_{\beta_{2}}+\left(H\left(\mu_{2}\right)-x\right) \omega_{\alpha_{2}}, \\
d e_{\alpha_{3}}= & \sum_{\beta_{3}} \omega_{\alpha_{3} \beta_{3}} e_{\beta_{3}}+\left(H\left(\mu_{3}\right)-x\right) \omega_{\alpha_{3}}, \\
d e_{n+1}= & -\mu_{1} \sum_{\alpha_{1}} \omega_{\alpha_{1}} e_{\alpha_{1}}-\mu_{2} \sum_{\alpha_{2}} \omega_{\alpha_{2}} e_{\alpha_{2}}-\mu_{3} \sum_{\alpha_{3}} \omega_{\alpha_{3}} e_{\alpha_{3}} .
\end{aligned}\right.
$$

From these equalities, we obtain

$$
\begin{aligned}
0=d^{2} e_{\alpha_{2}}= & \sum_{\beta_{2}} d \omega_{\alpha_{2} \beta_{2}} e_{\beta_{2}}-\sum_{\beta_{2}} \omega_{\alpha_{2} \beta_{2}} \wedge\left\{\sum_{\gamma 2} \omega_{\beta_{2} \gamma_{2}} e_{\gamma_{2}}+\left(H\left(\mu_{2}\right)-x\right) \omega_{\beta_{2}}\right\} \\
& +d\left(H\left(\mu_{2}\right)-x\right) \wedge \omega_{\alpha_{2}}+\left(H\left(\mu_{2}\right)-x\right) d \omega_{\alpha_{2}}
\end{aligned}
$$


i. e.

$$
\begin{gathered}
\sum_{\beta_{2}}\left(d \omega_{\alpha_{2} \beta_{2}}-\sum_{\gamma_{2}} \omega_{\alpha_{2} \gamma_{2}} \wedge \omega_{\gamma_{2} \beta_{2}}\right) e_{\beta_{2}}+\left(d \omega_{\alpha_{2}}-\sum_{\beta_{2}} \omega_{\alpha_{2} \beta_{2}} \wedge \omega_{\beta_{2}}\right)\left(H\left(\mu_{2}\right)-x\right) \\
+d\left(H\left(\mu_{2}\right)-x\right) \wedge \omega_{\alpha_{2}}=0
\end{gathered}
$$

and analogously

$$
\begin{gathered}
\sum_{\beta_{3}}\left(d \omega_{\alpha_{3} \beta_{3}}-\sum_{\gamma_{3}} \omega_{\alpha_{3} \gamma_{3}} \wedge \omega_{\gamma_{3} \beta_{3}}\right) e_{\beta_{3}}+\left(d \omega_{\alpha_{3}}-\sum_{\beta_{3}} \omega_{\alpha_{3} \beta_{3}} \wedge \omega_{\beta_{3}}\right)\left(H\left(\mu_{3}\right)-x\right) \\
+d\left(H\left(\mu_{3}\right)-x\right) \wedge \omega_{\alpha_{3}}=0 .
\end{gathered}
$$

Let $Y_{2}$ be any vector perpendicular to $E\left(\mu_{2}\right)$ and $H\left(\mu_{2}\right)-x$ at the point $x$, then we get from (6.8)

$$
\left\langle Y_{2}, d\left(H\left(\mu_{2}\right)-x\right)\right\rangle \wedge \omega_{\alpha_{2}}=0 .
$$

Since $\operatorname{dim} E\left(\mu_{2}, x\right) \geqq 2$, this equality implies

$$
\left\langle Y_{2}, d\left(H\left(\mu_{2}\right)-x\right)\right\rangle=0 \text {. }
$$

Hence, we have the following equality for the $\left(m_{2}+1\right)$-vector $\left(H\left(\mu_{2}\right)-x\right) \wedge e_{m_{1}+1}$ $\wedge \cdots \wedge e_{m_{1}+m_{2}}$ in $R^{n+2}$

$$
\begin{gathered}
d\left\{\left(H\left(\mu_{2}\right)-x\right) \wedge e_{m_{1}+1} \wedge \cdots \wedge e_{m_{1}+m_{2}}\right\} \\
=\frac{1}{\left|H\left(\mu_{2}\right)\right|^{2}+1}\left\langle d\left(H\left(\mu_{2}\right)-x\right), H\left(\mu_{2}\right)-x\right\rangle\left(H\left(\mu_{2}\right)-x\right) \wedge e_{m_{1}+1} \wedge \cdots \wedge e_{m_{1}+m_{2}}
\end{gathered}
$$

and analogously

$$
\begin{gathered}
d\left\{\left(H\left(\mu_{3}\right)-x\right) \wedge e_{m_{1}+m_{2}+1} \wedge \cdots \wedge e_{n}\right\} \\
=\frac{1}{\left|H\left(\mu_{3}\right)\right|^{2}+1}\left\langle d\left(H\left(\mu_{3}\right)-x\right), H\left(\mu_{3}\right)-x\right\rangle\left(H\left(\mu_{3}\right)-x\right) \wedge e_{m_{1}+m_{2}+1} \wedge \cdots \wedge e_{n} .
\end{gathered}
$$

(6.10) and (6.11) imply that there exist two fixed $\left(m_{2}+1\right)$-plane $E_{2}^{m_{2}+1}$ and $\left(m_{3}+1\right)-$ plane $E_{3}^{n_{3}+1}$ in $R^{n+2}$ through the origin such that

$$
\begin{aligned}
& \left(H\left(\mu_{2}\right)-x\right) \wedge e_{m_{1}+1} \wedge \cdots \wedge e_{m_{1}+m_{2}} / / E_{2}^{m_{2}+1}, \\
& \left(H\left(\mu_{3}\right)-x\right) \wedge e_{m_{1}+m_{2}+1} \wedge \cdots \wedge e_{n} / / E_{3}^{m_{3}+1} .
\end{aligned}
$$

Let $E_{\imath, x}^{m_{i}+1}$ be the $\left(m_{\imath}+1\right)$-plane through $x$ parallel to $E_{\imath}^{m_{i}+1}, \imath=2,3$. Then, we see easily that $E_{\imath, x}^{m_{i}+1} \cap S^{n+1}$ contains the integral submanifold of the distribution $E\left(\mu_{\imath}\right)$ through $x$. Since we have

$$
\begin{gathered}
x \wedge\left(H\left(\mu_{2}\right)-x\right) \wedge\left(H\left(\mu_{3}\right)-x\right)=x \wedge H\left(\mu_{2}\right) \wedge H\left(\mu_{3}\right) \\
=m_{1}\left(\frac{f-a_{2}}{m_{3}\left(F-n a_{3}\right)}+\frac{f-a_{3}}{m_{2}\left(F-n a_{2}\right)}\right) x \wedge \nabla f \wedge e_{n+1} \neq 0,
\end{gathered}
$$

the $\left(m_{2}+m_{3}+2\right)$-plane containing $E_{2, x}^{m_{2}+1}$ and $E_{3, x}^{m_{3}+1}$, which we denote by $E_{2,3, x}^{m_{2}+m_{3}+2}$, does not contain the origin of $R^{n+2}$. Furthermore, the small $\left(m_{2}+m_{3}+1\right)$-sphere 
$S_{2,3, x}^{m_{2}+m_{3}+1}=E_{2,3, x}^{m_{2}+m_{3}+2} \cap S^{n+1}$ contains the integral submanifold $K^{m_{2}+m_{3}}$ of the distribution $E\left(\mu_{2}\right)+E\left(\mu_{3}\right)$. Thus, we see that $K^{m_{2}+m_{3}}$ can be considered locally as a locus of moving $S_{2, x}^{m_{2}}=E_{2, x}^{m_{2}+1} \cap S^{n+1}$, where $x$ is a moving point along $S_{3, x_{0}}^{m_{3}}=E_{3, x_{0}}^{m_{3}+1} \cap S^{n+1}$.

On the other hand, from (6.6) we have

$$
\begin{aligned}
0=d \omega_{\alpha_{2} \alpha_{3}} & =\sum_{j=1}^{n} \omega_{\alpha_{2} \jmath} \wedge \omega_{j \alpha_{3}}+\omega_{\alpha_{2}(n+1)} \wedge \omega_{(n+1) \alpha_{3}}-\omega_{\alpha_{2}} \wedge \omega_{\alpha_{3}} \\
& =\sum_{\beta_{1}} \omega_{\alpha_{2} \beta_{1}} \wedge \omega_{\beta_{1} \alpha_{3}}-\left(\mu_{2} \mu_{3}+1\right) \omega_{\alpha_{2}} \wedge \omega_{\alpha_{3}} \\
& =-\left\{\frac{m_{1}^{2}}{\left(F-n a_{2}\right)\left(F-n a_{3}\right)} \sum_{\beta_{1}}\left(f_{\beta_{1}}\right)^{2}-\frac{\left(f-a_{2}\right)\left(f-a_{3}\right)}{m_{2} m_{3}}+1\right\} \omega_{\alpha_{2}} \wedge \omega_{\alpha_{3}},
\end{aligned}
$$

from which we get the equality for $f$

$$
\begin{array}{r}
\frac{m_{1}^{2}}{\left\{m_{1} f-\left(m_{1}+m_{3}\right) a_{2}+m_{3} a_{3}\right\}\left\{m_{1} f_{1}+m_{2} a_{2}-\left(m_{1}+m_{2}\right) a_{3}\right\}}|\nabla f|^{2} \\
-\frac{\left(f-a_{2}\right)\left(f-a_{3}\right)}{m_{2} m_{3}}+1=0
\end{array}
$$

i. e.

$$
\begin{aligned}
|\nabla f|^{2}= & \left\{f-a_{2}-\frac{m_{3}}{m_{1}}\left(a_{2}-a_{3}\right)\right\}\left\{f-a_{3}-\frac{m_{2}}{m_{1}}\left(a_{3}-a_{2}\right)\right\} \times \\
& \times\left\{\frac{\left(f-a_{2}\right)\left(f-a_{3}\right)}{m_{2} m_{3}}-1\right\} .
\end{aligned}
$$

Hence, we have

$$
\begin{aligned}
\left\langle H\left(\mu_{2}\right)-x, H\left(\mu_{3}\right)-x\right\rangle= & \frac{m_{1}^{2}}{\left(F-n a_{2}\right)\left(F-n a_{3}\right)}|\nabla f|^{2} \\
& -\frac{\left(f-a_{2}\right)\left(f-a_{3}\right)}{m_{2} m_{3}}+1=0,
\end{aligned}
$$

that is

$$
\left(H\left(\mu_{2}\right)-x\right) \perp\left(H\left(\mu_{3}\right)-x\right)
$$

and so

$$
E_{2}^{m_{2}+1} \perp E_{3}^{m_{3}+1} .
$$

We notice here that (6.12) will be reduced to (4.13), if we put $f=f_{1}, a_{2}=a_{3}=a$. And, by Lemma 11, any orthogonal trajectory $C$ of the level hypersurfaces of the function $f$ is an arc of a geodesic of $M^{n}$. C lies in an integral submanifold $K_{1}^{m_{1}}$ of the distribution $E\left(\mu_{1}\right)$.

Now, we prove the following 
THEOREM 5. Let $M^{n}$ be a minmmal hypersurface in $S^{n+1}$ as in Theorem 2 with $H\left(\mu_{i}\right) \neq 0, \imath=1,2,3$, then $d \mu_{\imath} \neq 0$ for $\imath=1,2,3$.

Proof. Let us suppose that $d \mu_{1} \equiv 0$, then $f_{2}$ and $f_{3}$ must be constants by Lemma 4. Setting $f_{2}=a_{2}$ and $f_{3}=a_{3}$, we have $a_{2} \neq a_{3}$ and $f_{1}=f$ is not a constant function by Lemma 12 . Thus, this must be the case (6.1) and so we can use the argument above in this section. We may consider as

$$
\begin{aligned}
& R^{n+2}=R_{1}^{m_{1}} \times R_{2}^{m_{2}+1} \times R_{3}^{m_{3}+1} \\
& R_{2}^{m_{2}+1}=E_{2}^{m_{2}+1}, \quad R_{3}^{m_{3}+1}=E_{3}^{m_{3}+1}, \quad R_{2} \approx R_{3} \approx R=R_{1}
\end{aligned}
$$

and we denote any point $x \in R^{n+2}$ as

$$
x=(\boldsymbol{y}, \boldsymbol{u}, \boldsymbol{v}), \quad \boldsymbol{y} \in R_{1}^{m_{1}}, \quad \boldsymbol{u} \in R_{2}^{m_{2}+1}, \quad \boldsymbol{v} \in R_{3}^{m_{3}+1} .
$$

Taking a fixed point $x_{0}=\left(\boldsymbol{y}_{0}, \boldsymbol{u}_{0}, \boldsymbol{v}_{0}\right) \in M^{n}$, we may assume that

$$
\begin{array}{ll}
R_{2}^{m_{2}+1}=R_{2}^{m_{2}} \times R_{2}, & R_{2}^{m_{2}} \| E^{m_{2}}\left(\mu_{2}, x_{0}\right), \\
R_{3}^{m_{3}+1}=R_{3}^{m_{3}} \times R_{3}, & R_{3}^{m_{3}} \| E^{m_{3}}\left(\mu_{3}, x_{0}\right) .
\end{array}
$$

Let $K_{x_{0}}^{m_{1}}$ be the integral submanifold of the distribution $E\left(\mu_{1}\right)$ through $x_{0}$ and $E_{1, x_{0}}^{m_{1}+1}$ the $\left(m_{1}+1\right)$-plane containing $K_{x_{0}}^{m_{1}}$. Since

$$
e_{1}, \cdots, e_{m_{1}}, H\left(\mu_{1}\right)-x_{0}=\mu_{1} e_{n+1}-x_{0} \| E_{1, x_{0}}^{m_{1}+1}
$$

we may consider as

$$
E_{1, x_{0}}^{m_{1}+1} \| R_{1}^{m_{1}} \times R_{2} \times R_{3}
$$

by (6.3) and (6.14). Therefore, $E_{1, x_{0}}^{m_{1}+1}$ is given by an equation such that

$$
\begin{gathered}
\sum_{\alpha_{1}=1}^{m_{1}} a_{\alpha_{1}} y_{a_{1}}+c_{2} u_{m_{2}+1}+c_{3} v_{m_{3}+1}=c_{0}, \\
u_{\alpha_{2}}=u_{0 \alpha_{2}}, \quad v_{\alpha_{3}}=v_{0 \alpha_{3}},
\end{gathered}
$$

where $a_{\alpha_{1}}, c_{2}, c_{3}$ and $c_{0}$ are real constants such that

$$
\sum_{\alpha_{1}=1}^{m_{1}} a_{\alpha_{1}}^{2}+c_{2}^{2}+c_{3}^{2}=1, \quad 0<c_{0}<1, c_{2}^{2}+c_{3}^{2} \neq 0
$$

and

$$
\boldsymbol{u}_{0}=\left(u_{01}, \cdots, u_{0 m_{2}}, u_{0\left(m_{2}+1\right)}\right), \quad \boldsymbol{v}_{0}=\left(v_{01}, \cdots, v_{0 m_{3}}, v_{0\left(m_{3}+1\right)}\right) \text {. }
$$

(Since $K_{x_{0}}^{m_{1}}$ is a small $m_{1}$-sphere of $S^{n+1}, c_{0}$ must be $0<\left|c_{0}\right|<1$ ). Therefore, for any point $x_{1}=\left(\boldsymbol{y}_{1}, \boldsymbol{u}_{1}, \boldsymbol{v}_{1}\right) \in K_{x_{0}}^{m_{1}}$ and any point $x=(\boldsymbol{y}, \boldsymbol{u}, \boldsymbol{v}) \in K_{x_{1}}^{m_{2}+m_{3}}$, we have

$$
\begin{aligned}
& \boldsymbol{y}=\boldsymbol{y}_{1} \\
& \langle\boldsymbol{u}, \boldsymbol{u}\rangle=\left\langle\boldsymbol{u}_{1}, \boldsymbol{u}_{1}\right\rangle=\sum_{\alpha_{2}=1}^{m_{2}}\left(u_{0 \kappa_{2}}\right)^{2}+\left(u_{1\left(m_{2}+1\right)}\right)^{2}
\end{aligned}
$$




$$
\langle\boldsymbol{v}, \boldsymbol{v}\rangle=\left\langle\boldsymbol{v}_{1}, \boldsymbol{v}_{1}\right\rangle=\sum_{\alpha_{3}=1}^{m_{3}}\left(v_{0 \alpha_{3}}\right)^{2}+\left(v_{1\left(m_{3}+1\right)}\right)^{2} .
$$

By a suitable change of orthogonal coordinates of $R_{1}^{m_{1}}$, we may put

$$
\boldsymbol{a}=\left(a_{1}, \cdots, a_{m_{1}}\right)=\left(c_{1}, 0, \cdots, 0\right) .
$$

Thus, setting $u_{1\left(m_{2}+1\right)}=t_{2}, v_{1\left(m_{3}+1\right)}=t_{3}$, we can represent locally and explicitly $M^{n}$ as :

$$
\left\{\begin{array}{l}
\langle\boldsymbol{y}, \boldsymbol{y}\rangle+\langle\boldsymbol{u}, \boldsymbol{u}\rangle+\langle\boldsymbol{v}, \boldsymbol{v}\rangle=1, \\
\langle\boldsymbol{u}, \boldsymbol{u}\rangle=t_{2}^{2}+b_{2}^{2},\langle\boldsymbol{v}, \boldsymbol{v}\rangle=t_{3}^{2}+b_{3}^{2}, \\
c_{1} y_{1}+c_{2} t_{2}+c_{3} t_{3}=c_{0}
\end{array}\right.
$$

where $b_{2}, b_{3}, c_{1}, c_{2}, c_{3}$ and $c_{0}$ are all constants such that

$$
0 \leqq b_{2} \leqq 1, \quad 0 \leqq b_{3} \leqq 1, \quad c_{1}^{2}+c_{2}^{2}+c_{3}^{2}=1, \quad 0<c_{0}<1
$$

and $t_{2}$ and $t_{3}$ are auxiliary variables.

Now, we compute the normal vector $M^{n}$ at $x$. By differentiating (6.16), we get

from which we obtain

$$
\begin{gathered}
\langle\boldsymbol{y}, d \boldsymbol{y}\rangle+\langle\boldsymbol{u}, d \boldsymbol{u}\rangle+\langle\boldsymbol{v}, d \boldsymbol{v}\rangle=0, \\
\langle\boldsymbol{u}, d \boldsymbol{u}\rangle=t_{2} d t_{2}, \quad\langle\boldsymbol{v}, d \boldsymbol{v}\rangle=t_{3} d t_{3}, \\
c_{1} d y_{1}+c_{2} d t_{2}+c_{3} d t_{3}=0,
\end{gathered}
$$

$$
d t_{2}=-\frac{1}{c_{3} t_{2}-c_{2} t_{3}}\left\langle c_{3} \boldsymbol{y}-t_{3} \boldsymbol{a}, d \boldsymbol{y}\right\rangle, \quad d t_{3}=\frac{1}{c_{3} t_{2}-c_{2} t_{3}}\left\langle c_{2} \boldsymbol{y}-t_{2} \boldsymbol{a}, d \boldsymbol{y}\right\rangle
$$

and, supposing $c_{3} t_{2}-c_{2} t_{3} \neq 0$, and

$$
\begin{aligned}
& \frac{t_{2}}{c_{3} t_{2}-c_{2} t_{3}}\left\langle c_{3} \boldsymbol{y}-t_{3} \boldsymbol{a}, d \boldsymbol{y}\right\rangle+\langle\boldsymbol{u}, d \boldsymbol{u}\rangle=0, \\
& -\frac{t}{c_{3} t_{2}-c_{2} t_{3}}\left\langle c_{2} \boldsymbol{y}-t_{2} \boldsymbol{a}, d \boldsymbol{y}\right\rangle+\langle\boldsymbol{v}, d \boldsymbol{v}\rangle=0 .
\end{aligned}
$$

Hence, the normal unit vector $N(x)$ at $x$ is represented as

$$
\begin{aligned}
N(x)= & \lambda\left(t_{2}\left(c_{3} \boldsymbol{y}-t_{3} \boldsymbol{a}\right),\left(c_{3} t_{2}-c_{2} t_{3}\right) \boldsymbol{u}, 0\right) \\
& +\mu\left(-t_{3}\left(c_{2} \boldsymbol{y}-t_{2} \boldsymbol{a}\right), 0,\left(c_{3} t_{2}-c_{2} t_{3}\right) \boldsymbol{v}\right) .
\end{aligned}
$$

Since $\langle x, N(x)\rangle=0$, we have

$$
\begin{aligned}
\lambda\left\{c_{3} t_{2}\langle\boldsymbol{y}, \boldsymbol{y}\rangle-t_{2} t_{3}\langle\boldsymbol{a}, \boldsymbol{y}\rangle+\left(c_{3} t_{2}-c_{2} t_{3}\right)\langle\boldsymbol{u}, \boldsymbol{u}\rangle\right\} \\
\quad+\mu\left\{-c_{2} t_{3}\langle\boldsymbol{y}, \boldsymbol{y}\rangle+t_{2} t_{3}\langle\boldsymbol{a}, \boldsymbol{y}\rangle+\left(c_{3} t_{2}-c_{2} t_{3}\right)\langle\boldsymbol{v}, \boldsymbol{v}\rangle\right\}=0 .
\end{aligned}
$$

The coefflcients of $\lambda$ and $\mu$ of the above equality are 


$$
c_{3}\left(1-b_{3}^{2}\right) t_{2}-c_{2} b_{2}^{2} t_{3}-c_{0} t_{2} t_{3} \text { and }-c_{2}\left(1-b_{2}^{2}\right) t_{3}+c_{3} b_{3}^{2} t_{2}+c_{0} t_{2} t_{3}
$$

respectively. Therefore, $N(x)$ is proportional to

$$
\begin{aligned}
& \left\{-c_{2}\left(1-b_{2}^{2}\right) t_{3}+c_{3} b_{3}^{2} t_{2}+c_{0} t_{2} t_{3}\right\}\left(t_{2}\left(c_{3} \boldsymbol{y}-t_{3} \boldsymbol{a}\right),\left(c_{3} t_{2}-c_{2} t_{3}\right) \boldsymbol{u}, 0\right) \\
+ & \left\{-c_{3}\left(1-b_{3}^{2}\right) t_{2}+c_{2} b_{2}^{2} t_{3}+c_{0} t_{2} t_{3}\right\}\left(-t_{3}\left(c_{2} \boldsymbol{y}-t_{2} \boldsymbol{a}\right), 0,\left(c_{3} t_{2}-c_{2} t_{3}\right) \boldsymbol{v}\right) \\
= & \left(c_{3} b_{3}^{2} t_{2}+c_{2} b_{2}^{2} t_{3}+c_{0} t_{2} t_{3}\right)\left(c_{3} t_{2}-c_{2} t_{3}\right)(\boldsymbol{y}, \boldsymbol{u}, \boldsymbol{v}) \\
- & \left(c_{3} t_{2}-c_{2} t_{3}\right)\left(t_{2} t_{3} \boldsymbol{a}, c_{2} t_{3} \boldsymbol{u}, c_{3} t_{2} \boldsymbol{v}\right) .
\end{aligned}
$$

Hence, supposing $t_{2} t_{3} \neq 0, N(x)$ is proportional to

$$
\begin{gathered}
\tilde{N}(x)=\left(c_{0}+\frac{b_{2}^{2} c_{2}}{t_{2}}+\frac{b_{3}^{2} c_{3}}{t_{3}}\right)(\boldsymbol{y}, \boldsymbol{u}, \boldsymbol{v})-\left(\boldsymbol{a}, \frac{c_{2}}{t_{2}} \boldsymbol{u}, \frac{c_{3}}{t_{3}} \boldsymbol{v}\right), \\
x=(\boldsymbol{y}, \boldsymbol{u}, \boldsymbol{v}) .
\end{gathered}
$$

Now, we compute the 1st and 2nd fundamental forms of $M^{n}$ at $x$, where $t_{2} \neq 0, t_{3} \neq 0$. From the above argument, we may still put

$$
\boldsymbol{u}=\left(0, \cdots, 0, p_{2}\right), \quad \boldsymbol{v}=\left(0, \cdots, 0, p_{3}\right)
$$

by changing the coordinate axises of $R_{2}^{m_{2}+1}$ and $R_{3}^{m_{3}+1}$. We get easily

$$
d u_{m_{2}+1}=\frac{t_{2}}{p_{2}} d t_{2}, \quad d v_{m_{3}+1}=\frac{t_{3}}{p_{3}} d t_{3}
$$

and

$$
\begin{aligned}
& d t_{2}=-\frac{1}{c_{3} t_{2}-c_{2} t_{3}}\left\{c_{3}\langle\boldsymbol{y}, d \boldsymbol{y}\rangle-c_{1} t_{3} d y_{1}\right\} \\
& d t_{3}=\frac{1}{c_{3} t_{2}-c_{2} t_{3}}\left\{c_{2}\langle\boldsymbol{y}, d \boldsymbol{y}\rangle-c_{1} t_{2} d y_{1}\right\} .
\end{aligned}
$$

Hence we have

$$
\begin{aligned}
d s^{2}= & \langle d \boldsymbol{y}, d \boldsymbol{y}\rangle+\langle d \boldsymbol{u}, d \boldsymbol{u}\rangle+\langle d \boldsymbol{v}, d \boldsymbol{v}\rangle \\
= & {\left[\langle d \boldsymbol{y}, d \boldsymbol{y}\rangle+\left(d u_{m_{2}+1}\right)^{2}+\left(d v_{m_{3}+1}\right)^{2}\right] } \\
& +\sum_{\alpha_{2}=1}^{m_{2}} d u_{\alpha_{2}} d u_{\alpha_{2}}+\sum_{\alpha_{3}=1}^{m_{3}} d v_{\alpha_{3}} d v_{\alpha_{3}} .
\end{aligned}
$$

By means of (6.17), setting $\varphi=c_{0}+\frac{b_{2}^{2} c_{2}}{t_{2}}+\frac{b_{3}^{2} c_{3}}{t_{3}}$, we have

$$
\begin{aligned}
& -\left\langle d^{2} x, \tilde{N}(x)\right\rangle=\langle d x, d \tilde{N}(x)\rangle=\varphi\langle d x, d x\rangle \\
& -\left\langle d \boldsymbol{u}, \frac{c_{2}}{t_{2}} d \boldsymbol{u}-\frac{c_{2}}{t_{2}^{2}} \boldsymbol{u} d t_{2}\right\rangle+\left\langle d \boldsymbol{v}, \frac{c_{3}}{t_{3}} d \boldsymbol{v}-\frac{c_{3}}{t_{3}^{2}} \boldsymbol{v} d t_{3}\right\rangle
\end{aligned}
$$




$$
=\varphi\langle d \boldsymbol{y}, d \boldsymbol{y}\rangle+\left(\varphi-\frac{c_{2}}{t_{2}}\right)\langle d \boldsymbol{u}, d \boldsymbol{u}\rangle+\left(\varphi-\frac{c_{3}}{t_{3}}\right)\langle d \boldsymbol{v}, d \boldsymbol{v}\rangle+\frac{c_{2}}{t_{2}} d t_{2} d t_{2}+\frac{c_{3}}{t_{3}} d t_{3} d t_{3}
$$

i. e.

$$
\begin{aligned}
-\left\langle d^{2} x, \tilde{N}(x)\right\rangle= & {\left[\varphi\langle d \boldsymbol{y}, d \boldsymbol{y}\rangle+\left(\varphi-\frac{c_{2}}{t_{2}}+\frac{c_{2} p_{2}^{2}}{t_{2}^{3}}\right)\left(d u_{m_{2}+1}\right)^{2}\right.} \\
& \left.+\left(\varphi-\frac{c_{3}}{t_{3}}+\frac{c_{3} p_{3}^{2}}{t_{3}^{3}}\right)\left(d v_{m_{3}+1}\right)^{2}\right] \\
& +\left(\varphi-\frac{c_{2}}{t_{2}}\right) \sum_{\alpha_{2}=1}^{m_{2}} d u_{\alpha_{2}} d u_{\alpha_{2}}+\left(\varphi-\frac{c_{3}}{t_{3}}\right) \sum_{\alpha_{3}=1}^{m_{3}} d v_{\alpha_{3}} d v_{\alpha_{3}} .
\end{aligned}
$$

(6.18) shows that the parameter submanifolds corresponding to $\left(y_{1}, \cdots, y_{m_{1}}\right)$, $\left(u_{1}, \cdots, u_{m_{2}}\right)$ and $\left(v_{1}, \cdots, v_{m_{3}}\right)$ are orthogonal to each others at $x$. And (6.19) show that the tangent spaces to the submanifolds corresponding to $\left(u_{1}, \cdots, u_{m_{2}}\right)$ and $\left(v_{1}, \cdots, v_{m_{3}}\right)$ must be $E\left(\mu_{2}, x\right)$ and $E\left(\mu_{3}, x\right)$ respectively. Therefore, the form in the brackets of (6.19) must be proportional to the one of (6.18).

Since we have

$$
\begin{gathered}
\langle d \boldsymbol{y}, d \boldsymbol{y}\rangle+\left(d u_{m_{2}+1}\right)^{2}+\left(d v_{m_{3}+1}\right)^{2} \\
=\langle d \boldsymbol{y}, d \boldsymbol{y}\rangle+\left(\frac{t_{2} t_{3}}{\left(c_{3} t_{2}-c_{2} t_{3}\right) p_{2} p_{3}}\right)^{2}\left[p_{2}^{2}\left(c_{1} d y_{1}-\frac{c_{2}}{t_{2}}\langle\boldsymbol{y}, d \boldsymbol{y}\rangle\right)^{2}\right. \\
\left.+p_{3}^{2}\left(c_{1} d y_{1}-\frac{c_{2}}{t_{3}}\langle\boldsymbol{y}, d \boldsymbol{y}\rangle\right)^{2}\right]
\end{gathered}
$$

and

$$
\begin{aligned}
& \varphi\langle d \boldsymbol{y}, d \boldsymbol{y}\rangle+\left(\varphi-\frac{c_{2}}{t_{2}}+\frac{c_{2} p_{2}^{2}}{t_{2}^{3}}\right)\left(d u_{m_{2}+1}\right)^{2}+\left(\varphi-\frac{c_{3}}{t_{3}}+\frac{c_{3} p_{3}^{2}}{t_{3}^{3}}\right)\left(d v_{m_{3}+1}\right)^{2} \\
= & \varphi\left\{\langle d \boldsymbol{y}, d \boldsymbol{y}\rangle+\left(d u_{m_{2}+1}\right)^{2}+\left(d v_{m_{3}+1}\right)^{2}\right\} \\
& +\left(\frac{t_{2} t_{3}}{\left(c_{3} t_{2}-c_{2} t_{3}\right) p_{2} p_{3}}\right)^{2}\left[\frac{b_{2}^{2} c_{2} p_{2}^{2}}{t_{2}^{3}}\left(c_{1} d y_{1}-\frac{c_{2}}{t_{2}}\langle\boldsymbol{y}, d \boldsymbol{y}\rangle\right)^{2}\right. \\
& \left.+\frac{b_{3}^{2} c_{3} p_{3}^{2}}{t_{3}^{3}}\left(c_{1} d y_{1}-\frac{c_{3}}{t_{3}}\langle\boldsymbol{y}, d \boldsymbol{y}\rangle\right)^{2}\right],
\end{aligned}
$$

the differential form in $d y_{1}, \cdots, d y_{m}$

$$
\frac{b_{2}^{2} c_{2} p_{2}^{2}}{t_{2}^{3}}\left(c_{1} d y_{1}-\frac{c_{2}}{t_{2}}\langle\boldsymbol{y}, d \boldsymbol{y}\rangle\right)^{2}+\frac{b_{3}^{2} c_{3} p_{3}^{2}}{t_{3}^{3}}\left(c_{1} d y_{1}-\frac{c_{3}}{t_{3}}\langle\boldsymbol{y}, d \boldsymbol{y}\rangle\right)^{2}
$$

must be proportional to (6.20). This is generally impossible. Thus, we reach a contradiction. 


\section{REFERENCES}

[1] T. Otsuki, A theorey of Riemannian manıfolds, Kōdaı Math. Sem. Rep., 20 (1968), 282-295.

[2] T. Otsuki, Pseudo-umbilical submanıfolds with M-1ndex $\leqq 1$ in Euclidean spaces, Kōdai Math. Sem. Rep., 20 (1968), 296-304.

[3] T. Otsuki, Minımal hypersurfaces in a Riemannian manıfold of constant curvature, Amer. J. Math., 112 (1970), 145-173.

Department of Mathematics

Tokyo Institute of Technology 